\title{
PROGRESS IN MODELLING FAST-ION D-ALPHA SPECTRA AND NEUTRAL PARTICLE ANALYZER FLUXES USING FIDASIM
}

B. Geiger ${ }^{1}$, L. Stagner ${ }^{2}$, W.W. Heidbrink ${ }^{3}$, R. Dux ${ }^{4}$, R. Fischer ${ }^{4}$, Y. Fujiwara ${ }^{5}$, A.V. Garcia $^{3}$, A. S. Jacobsen ${ }^{6}$, A. Jansen van Vuuren ${ }^{4}$, A. N. Karpushov ${ }^{7}$, D. Liu ${ }^{3}$, P. A. Schneider $^{4}$, I. Sfiligoi ${ }^{8}$, P. Zs. Poloskei ${ }^{9}$, and M. Weiland ${ }^{4}$

${ }^{1}$ University of Wisconsin, Madison, USA

${ }^{2}$ Oak Ridge Institute for Science and Education, Oak Ridge, TN, USA

${ }^{3}$ University of California, Irvine, CA, USA

${ }^{4}$ Max-Planck-Institute for Plasma Physics, Boltzmannstr. 2, 85748 Garching, Germany

${ }^{5}$ National Institute for Fusion Science, Toki, Japan

${ }^{6}$ Culham Centre for Fusion Energy, Culham, England

7 Ecole Polytechnique Fédérale de Lausanne (EPFL), Swiss Plasma Center (SPC), CH1015 Lausanne, Switzerland

${ }^{8}$ San Diego Supercomputer Center, La Jolla, California 92093, US

${ }^{9}$ Max-Planck-Institute for Plasma Physics, Greifswald, Germany

Email of corresponding author: benedikt.geiger@wisc.edu

\begin{abstract}
FIDASIM is a code that models signals produced by charge-exchange reactions between neutrals and ions (both fast and thermal) in magnetically confined plasmas. With the ion distribution function as input, the code predicts the efflux to a neutral particle analyzer (NPA) diagnostic and the photon radiance of Balmer-alpha light to a fast-ion $\mathrm{D}_{\alpha}$ (FIDA) diagnostic, in addition to many other related quantities. A new, parallelized version of the Monte Carlo code FIDASIM has been developed in Fortan90 that is substantially faster than the original IDL version. Modified algorithms include more accurate treatments of the time dependent collisional-radiative equations that describe neutral energy levels, of the cloud of "halo" neutrals that surround the injected neutral beam, and of finite Larmor radius effects. Enhanced physics capabilities include modeling "passive" signals from cold edge neutrals, the ability to treat general three-dimensional magnetic confinement configurations, and calculations of diagnostic-specific weight functions that enable tomographic reconstructions of the fast-ion distribution function. Neutral beam attenuation, beam emission, and fast-ion birth profiles are also modelled. The new algorithms have been successfully validated against experimental data and new features have been tested through benchmarks between two independently developed versions of the code.
\end{abstract}




\section{Introduction}

In magnetically confined fusion plasmas, diagnosing super-thermal populations of energetic ions is important, as these "fast ions" are used to heat the plasma and drive current but, if unconfined, can damage vacuum vessel components. For hydrogenic fast ions, chargeexchange reactions with neutrals provide an important source of information about the confined fast-ion population. Neutral particle analyzers (NPA) measure fast neutrals that escape from the plasma following a charge-exchange reaction. Fast-ion D-alpha (FIDA) diagnostics [1] measure the Balmer-alpha light that is emitted by neutralized fast ions. The first NPA diagnostics [2] used the ambient population of neutrals to provide donor electrons for the charge-exchange reaction; these reactions with cold neutrals are called "passive" charge exchange. However, injected neutral beams are required in present day experiments to diagnose the core with good spatial resolution since the density of ambient neutrals is typically low in high temperature and large-scale plasmas; reactions with injected neutrals are called "active" charge exchange.

For the interpretation of such active charge exchange signals, the synthetic diagnostic code FIDASIM was developed which models the density of neutrals along neutral beam injection (NBI) and provides synthetic FIDA spectra and NPA fluxes based on input fastion distribution functions. The first version of FIDASIM has been written in the Interactive Data Language (IDL) and is described in [3].

Since that early publication, FIDASIM has been improved and extended significantly. Now, FIDASIM is a parallelized Fortran90 code with Python and IDL interfaces that works equally well for hydrogen neutrals (not only deuterium) and additionally allows to predict passive signals. It has been equipped with an updated collisional radiative model and a novel approach simulating the population of halo neutrals arising from charge exchange reactions of thermal ions along a given NBI path. This made FIDASIM a valuable tool, not only for fast-ion studies, but also for the interpretation of main ion measurements $[4,5,6]$ and for the calculation of impurity densities from charge exchange recombination (CER) spectroscopy measurements [7]. In addition, FIDASIM has been equipped with a new and more efficient algorithm to calculate NPA fluxes and provides various new outputs such as "weight functions" of FIDA diagnostics or fast-ion birth profiles. The basic structure of the code is displayed in Fig. 1, showing an updated version of the previously published flow diagram in [3]. After the initialization and collection of input data, the density of beam neutrals along the path of a given diagnostic beam source is calculated. This is done by tracking Monte Carlo markers (representing neutrals) through a 3D simulation grid and calculating their attenuation and excitation due to collisions with the background plasma. The resulting beam density is then used to calculate the charge exchange probability of thermal ions such that so-called direct charge exchange (DCX) neutrals can be tracked through the simulation grid. Next, the charge exchange probability between DCX neutrals and thermal ions is determined, allowing the simulation of the halo 
neutral population surrounding NBI. Alternatively to this calculation of beam, DCX and halo neutral densities, a background (cold) neutral population can be specified as input - as needed for passive simulations. Finally, either FIDA or NPA spectra are calculated which require the previously mentioned neutral densities, as well as a predicted fast-ion distribution function or particle distribution as input. In each of these steps, spectra for pre-defined lines of sight can be determined. This allows FIDASIM to produce Balmer alpha spectra consisting of the beam emission, the thermal charge-exchange component (DCX+halo) and the FIDA emission. All stages of the code have seen changes while the most significant ones, affecting the physics model, are highlighted in red in Fig. 1.

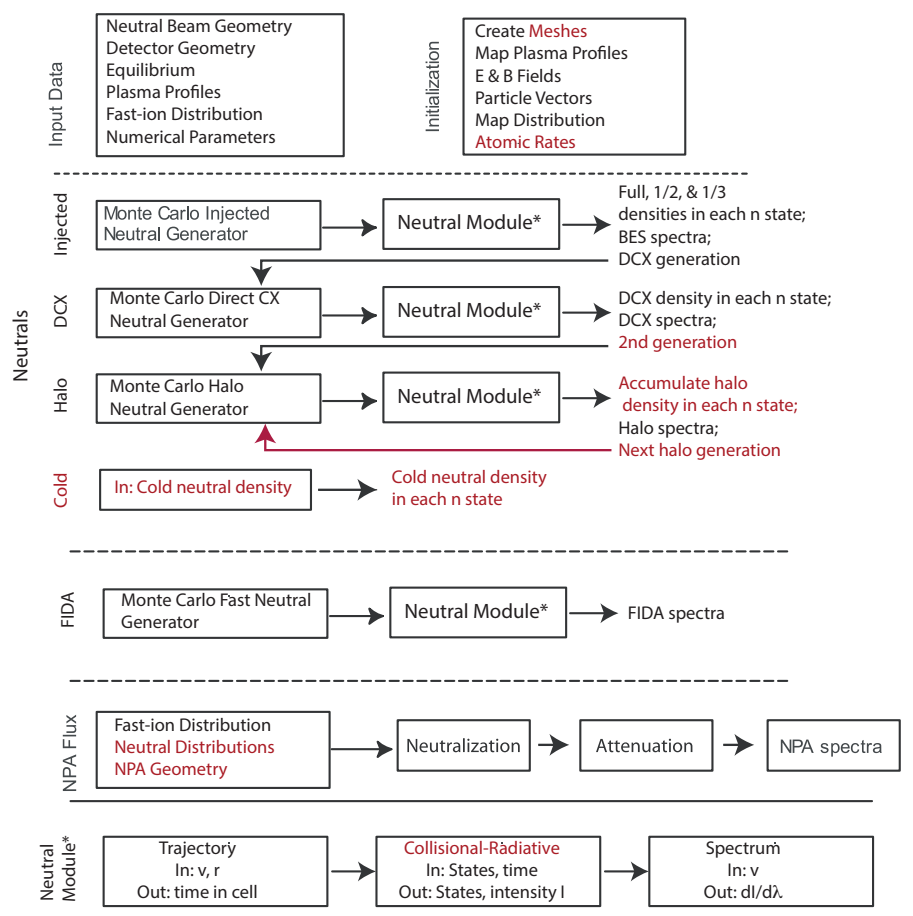

Figure 1: Flow diagram for the FIDASIM code. Portions labeled in red have undergone significant modification from the original version.

The purpose of this paper is to document these changes compared to the original IDL version of FIDASIM [3]. The most important modifications will therefore be introduced in section 2: The updated collisional radiative model, the new method of computing halo neutrals, a new and more efficient NPA simulation module and additional output are discussed. Moreover, verification and validation are crucial for any computer code. Several validation cases and tests are thus presented in section 3 . After the conversion of FIDASIM to FORTRAN in 2013, separate versions of the code were developed in the USA and EU with similar new features and functionality being introduced. Beginning in 2018, we decided 
to reunify FIDASIM into a single, well-supported, international version available via [8]. A set of comparison benchmarks of the two versions were completed and are described in section 4. The paper concludes with the outlook for future code enhancements (Sec. 5). Finally, it should be noted that the Appendix documents additional key components of the code such the required inputs (Appendix A), the applied atomic cross sections and rates (Appendix F) or the definition of the 3D modelling grid (Appendix B).

\section{Updates of the physics model}

\subsection{New collisional radiative model}

The collisional radiative model (named colrad) is the core-element of FIDASIM. colrad is considered along each step of a given Monte Carlo marker's path through the 3D spatial simulation grid of FIDASIM. It solves the time-dependent collisional-radiative equations and calculates the ionization and excitation, de-excitation, and photon emission of neutrals represented by markers.

The markers represent a flux $\Gamma$ of hydrogenic neutrals in atomic energy-level states with principal quantum numbers between $n=1$ and $n=6$. In each cell of the 3D simulation grid, the evolution of the atomic state population is calculated, depending on the local plasma parameters (densities, temperatures, and rotation). The collisional radiative model considers the finite lifetime of excited states and takes collision-induced de-excitation into account. The evolution of atomic states, $n$, of a given neutral is described by the following system of first order linear ordinary differential equations:

$$
\left(\begin{array}{c}
d \Gamma_{1} / d t \\
d \Gamma_{2} / d t \\
\vdots \\
d \Gamma_{6} / d t
\end{array}\right)=\left(\begin{array}{cccc}
a_{11} & a_{12} & \ldots & a_{16} \\
a_{21} & a_{22} & \ldots & a_{26} \\
\vdots & \vdots & \ddots & \vdots \\
a_{61} & a_{62} & \ldots & a_{66}
\end{array}\right) \quad\left(\begin{array}{c}
\Gamma_{1} \\
\Gamma_{2} \\
\vdots \\
\Gamma_{6}
\end{array}\right)
$$

Here, $\Gamma_{n}$ in $[1 / \mathrm{s}]$ is the flux of neutrals in the state $n$ and $d \Gamma_{n} / d t$ is its derivative. The matrix $\mathbf{A}$ consists of rates, $a_{n m}$ in $[1 / \mathrm{s}]$ that describe the ionization (including charge exchange), excitation, and de-excitation of neutrals. The rates are stored in pre-calculated look-up tables that depend on the neutral's (marker's) energy, the ambient temperature, $n$ and $m$. The corresponding cross-sections, as well as the approach to calculate the rates, are described in detail in Appendix F. Note here that almost all cross-sections and rates saw important updates. Moreover, the rates are now stored as a function of the neutral's energy $E$ per atomic mass unit and the ion temperature per atomic mass unit of the background plasma. By further considering the atomic masses in colrad, FIDASIM became capable of simulating hydrogen or tritium plasmas equally well as deuterium ones. Moreover, care

has been taken in extending the cross-sections to low energies such that it is now possible to simulate signals from thermal distribution functions. 
When applying the collisional radiative model in FIDASIM, first, the different matrix elements are determined. The off-diagonal elements $a_{n m}$ are defined positively and account for the populating transitions from an initial principal quantum state $n$ to a final principal quantum state $m$. The off-axis elements represent the sum of $k$ individual rates, $c_{n m}(k)$ for electron-, ion-, and impurity-impact excitation and de-excitation and for spontaneous de-excitation (the Einstein coefficients):

$$
a_{n m}=\sum_{k} c_{n m}(k)
$$

The diagonal elements are defined negatively and correspond to the depopulating transitions. They depend on the sum of the $k$ individual excitation and de-excitation processes into all final states other than $n$ and the $j$ different loss mechanisms $l_{n}(j)$ that are caused by impact ionization by electrons, hydrogen, and impurities and charge exchange with hydrogen ions and impurities

$$
a_{n n}=-\sum_{k} \sum_{m=1}^{6} c_{n m}(k)-\sum_{j} l_{n}(j) .
$$

While cross-sections have been determined up to $\mathrm{n}=12$, FIDASIM only retains energy levels up to $n=6$ for the sake of reduced computational effort. Impact excitation into states higher than $n=6$ is therefore summed up and considered as a loss mechanism. Owing to high cross-sections for impact ionization and charge exchange for these states, neutrals that are excited into high-energy states are quickly lost [9].

Having created the matrix $\mathbf{A}$, the flux of neutrals $\boldsymbol{\Gamma}\left(t_{0}+d t\right)$ after a time interval, $d t$, is determined by solving Eq. 1. Previously, a fourth order Runge Kutta method was used that has been replaced by an analytic solution to eliminate numerical instabilities. The analytic method has been developed according to [10] and is based on the determination of the eigenvectors, $\overrightarrow{s_{i}}$, and the eigenvalues, $\lambda_{i}$, of matrix $\mathbf{A}$ which fulfill:

$$
\mathbf{A} \cdot \overrightarrow{s_{i}}=\overrightarrow{s_{i}} \cdot \lambda_{i}
$$

The analytic solution of Eq. 1 takes the form of a matrix exponential,

$$
\boldsymbol{\Gamma}\left(t_{0}+d t\right)=\left(\left(\boldsymbol{\Gamma}\left(t_{0}\right) \times \mathbf{S}^{-1}\right) e^{\boldsymbol{\Lambda} d t}\right) \times \mathbf{S}
$$

where $\boldsymbol{\Gamma}(t)$ is a vector of the individual fluxes $\Gamma_{i}$, " $\times$ " stands for matrix multiplication, $\mathbf{S}$ is the matrix of the individual eigenvectors $\overrightarrow{s_{i}}$, and $\boldsymbol{\Lambda}$ is a diagonal matrix containing the eigenvalues $\lambda_{i}$. The number of particles $\mathbf{N}$ in the various n-levels represented by a given marker within the time-range $d t$ (i.e, the integral $\boldsymbol{\Gamma}$ ) can be determined using:

$$
\mathbf{N}=\left(\left(\boldsymbol{\Gamma}\left(t_{0}\right) \times \mathbf{S}^{-1}\right)\left(e^{\boldsymbol{\Lambda} d t}-1\right) / \boldsymbol{\Lambda}\right) \times \mathbf{S}
$$


By dividing $\mathbf{N}$ with a given cell's volume and accounting for the marker's weight, the neutral density contribution is obtained. If requested, the Balmer alpha light emitted by a given maker is calculated by multiplying the $n=3$ component of the density with the Einstein coefficient for spontaneous de-excitation from $n=3$ to $n=2$.

Verification of this new approach of the collisional-radiative model is discussed in Sec. 3.

\subsection{New halo model}
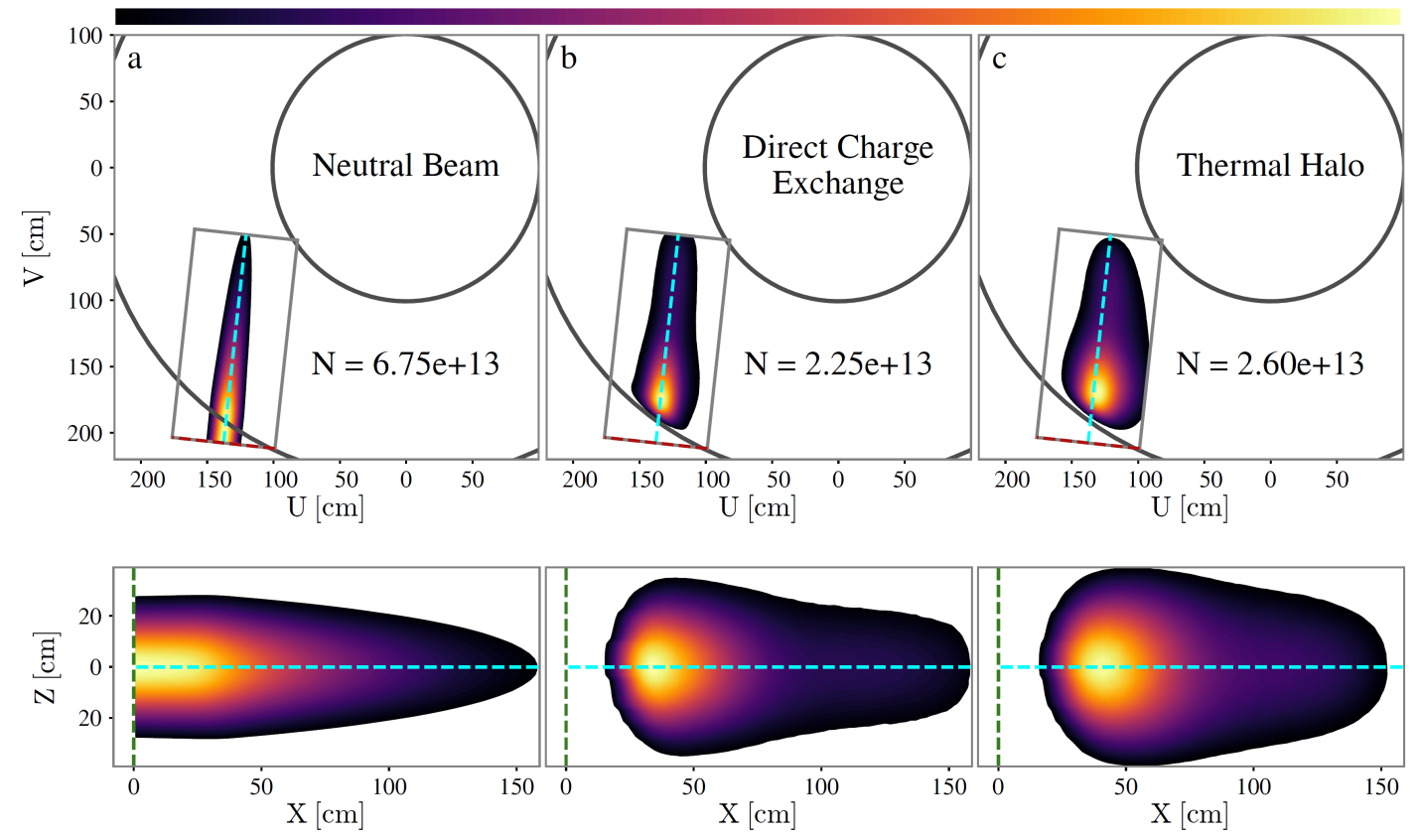

Figure 2: Profiles of (a) injected beam, (b) direct charge exchange (DCX), and (c) thermal halo neutrals. Top row: 2D profiles in machine coordinates, integrated over elevation $Z$. Bottom row: 2D profiles in beam grid coordinates that are aligned with the beam, integrated over the horizontal dimension $Y$. The beam grid axes are color coded: $X$ axis (dashed cyan), $Y$ axis (dashed red), and $Z$ axis (dashed green). The number of neutrals in each population is listed. The color map on the top increases from left to right. The beam geometry is the 210RT neutral beam on DIII-D.

FIDASIM calculates $n$-level resolved neutral densities for various neutral populations: Injected neutrals at the full, half, and one-third energies, halo (+direct charge exchange) neutrals, cold neutrals, and fast neutrals. While the calculations of injected and fast neutrals are described in Appendices $\mathrm{C}$ and $\mathrm{E}$ (no major changes were made), the simulation of halo neutrals has seen a major modification. Rather than a random walk algorithm, 
the new approach utilizes multiple generations of neutrals arising from thermal chargeexchange reactions. The process begins when beam neutrals undergo charge exchange with thermal ions, creating a new neutral population, the DCX neutrals. Monte Carlo ions are selected from the thermal-ion population and the probability of charge exchange with the injected neutrals is computed. After their birth (often in an excited $n$ level), DCX neutrals travel ballistically and the energy-level populations evolve in accordance with the collisional radiative model, producing Balmer- $\alpha$ light along the way. The DCX density and emission produced by a cell is determined by summing the contributions from each DCX trajectory. The total DCX density is calculated by repeating the above process for every cell that contains beam neutrals.

Likewise, the DCX neutrals can also undergo charge exchange with the thermal ions. The process of a neutral population charge-exchanging with the thermal ions can repeat $a d$ infinitum; the overall effect is a thermal halo of neutrals surrounding the neutral beam. The iterative process is demonstrated in Eq. 7.

$$
\begin{array}{rll}
\text { DCX }: & & H_{t h}^{+}+H_{N B I} \rightarrow H_{D C X}+H_{N B I}^{+} \\
\text {Halo }: & & H_{t h}^{+}+H_{D C X} \rightarrow H_{\text {halo }(0)}+H_{t h}^{+} \\
\text {Halo }: & & H_{t h}^{+}+H_{\text {halo }(0)} \rightarrow H_{\text {halo }(1)}+H_{t h}^{+} \\
& \vdots & \\
\text { Halo }: & & H_{\text {th }}^{+}+H_{\text {halo }(i-1)} \rightarrow H_{\text {halo }(i)}+H_{t h}^{+}
\end{array}
$$

Here, $H_{N B I}$ represents the initial injected neutral, $H_{D C X}$ is the first-generation (DCX) neutral, and $H_{h a l o(i)}$ is the $i$ th generation of halo neutrals. From generation to generation, the flux of halo neutrals decreases compared to the previous generation because more and more neutrals are lost through electron-, ion-, and impurity-impact ionization, processes that are accounted for in the collisional-radiative model. Consequently, the iteration over halo generations is guaranteed to converge. The process for calculating the halo densities and emission is similar to the DCX calculation, just repeated until the amount of halo neutrals produced in a generation is $1 \%$ of the initial seed population, which is the DCX neutrals. The main difference between the halo and the DCX calculation is the velocity distribution of the reactants. When calculating the charge-exchange probability to form a given DCX neutral, the relative velocity between the injected neutrals $H_{N B I}$ and a given thermal ion $H_{t h}$ is considered. In the case of halo neutrals we assume that $H_{D C X}$ and $H_{\text {halo }(i-1)}$ have a thermal velocity distribution.

Examples of the injected, DCX, and halo neutral density profiles are shown in Fig. 2. The injected neutral density steadily attenuates, while the DCX and halo profiles peak where the attenuation rate is large. As expected, owing to broadening associated with the random thermal-ion velocities, the DCX profile is broader than the injected neutral profile. Similarly, the halo profile is broader than the DCX profile, since the additional generations 
have more opportunity to diffuse.
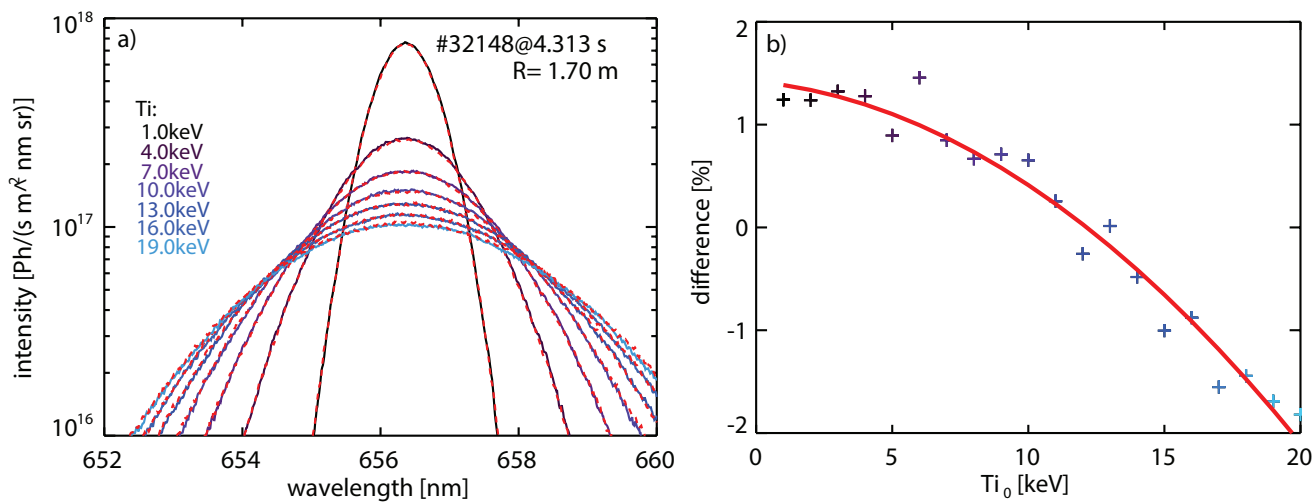

Figure 3: a) Predicted halo spectra for artificially modified ion temperatures in AUG discharge \#32148. In FIDASIM, either a Maxwell distribution of halo and DCX neutrals (method 1, red-dashed) was assumed or stored velocity vectors of the i-th generation were considered to calculate the neutralization probability of generation $\mathrm{i}+1$ (method 2 , black/blue). b) Relative difference between halo spectra predicted using methods 1 and 2 as a function of the considered central ion temperature.

The assumption of a thermal velocity distribution halo and DCX neutrals can be justified as the cross-sections for charge exchange do not exhibit a very strong energy dependence at thermal collision energies (see cross-sections in Fig. 18). In addition, the assumption has been checked by storing velocity vectors of neutrals of each generation to calculate the charge exchange probability of the next generation. Fig. 3a compares calculated halo emission intensities as a function of wavelength assuming either a Maxwell distribution of halo and DCX neutrals (method 1) or considering the stored velocity vectors of halo neutrals (method 2). The halo spectra have been calculate for a representative ASDEX Upgrade deuterium plasma (\#32148) with 7.5 MW of NBI heating power, about $2 \mathrm{MW}$ of ECRH power, a line-average density of $5 \times 10^{19} / \mathrm{m}^{3}$ and a core electron temperature of $\sim 6 \mathrm{keV}$. Simulations have been performed for 20 different ion-temperature profiles that have been scaled to reach central values between $1 \mathrm{keV}$ and $20 \mathrm{keV}$. The difference between the resulting halo spectra considering the two methods is very small. This can also be seen in Fig. 3b which displays the relative difference between the two methods: $\left(I_{1}-I_{2}\right) / I_{1} * 100$ where $I_{1}$ and $I_{2}$ are the total simulated halo intensities from methods 1 and 2, respectively. The relative differences are below $2 \%$ and tend to increase towards high temperatures which can be explained since the cross-section for charge exchange strongly decreases at large collision energies (see Fig. 18). Note here that the simulation data shows some random behaviour which can be attributed to Monte Carlo noise. 


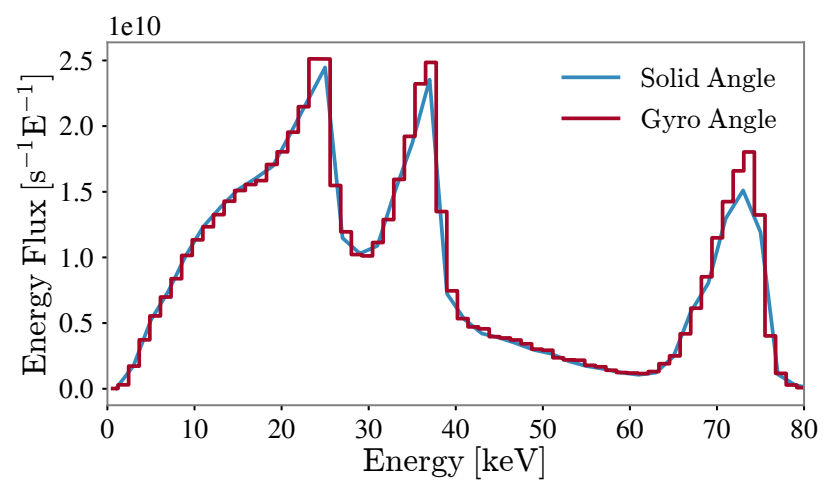

Figure 4: Incident energy flux for the DIII-D imaging NPA: Solid-angle NPA calculation (blue) and the gyroangle approach (red). Both methods give the same simulated spectrum.

\section{$2.3 \quad$ NPA flux}

Neutral particle analyzers collect fast neutrals that escape the plasma. However, since most fast-neutral trajectories miss the detectors, a brute force approach as used in the early FIDASIM version that randomly launches neutrals from within the plasma is extremely inefficient. Rather, the present version of FIDASIM utilizes the detector geometry to restrict calculations to trajectories that are accepted by a detector.

Since FIDASIM accepts either fast-ion distribution functions or Monte Carlo particle distributions as input (see Appendix A), two different approaches are being used. For fast-ion distribution functions, the spatial region from which neutrals can be detected by a given NPA is determined by checking whether the trajectories from a specified plasma position to a point on the detector pass through the aperture. If they do, the pitch $p$ of the fast neutral is calculated. With the fast-ion velocity $\mathbf{v}$ known, the total $n$-level weighted charge exchange probability with the various neutral populations $p_{C X}$ is computed. The emitted neutral is known on the full Lorentz orbit so, to evaluate the fast-ion distribution function, a gyrostep is taken to find $F(E, p)$ at the guiding center. To calculate the attenuation of escaping neutrals, the collisional radiative model is then solved along the neutral trajectory, yielding the probability $p_{\text {atten }}$ of reaching the detector without ionizing. This position contributes a signal proportional to $F(E, p) p_{\Omega} p_{C X} p_{\text {atten }} V_{\text {cell }}$ to the NPA flux, where $V_{\text {cell }}$ is the volume of the grid cell and $p_{\Omega}$ the geometrical probability to reach the detector. The calculation sums over all cells with possible trajectories into the detector. As an example, Fig. 4 shows in blue the energy spectrum of neutrals incident on the stripping foil for DIII-D's imaging NPA diagnostic [11].

For Monte Carlo distributions we analytically find the range of gyroangles that intersect 
the NPA detector, rather than calculating solid angles. This algorithm assumes that the magnetic field does not change substantially over a Larmor radius, so the gyroradius is a constant, forming a ring around the guiding center. Neutrals emitted from this ring with the correct pitch $p$ and gyroangle $\gamma$ will reach the detector. For a given pitch, the emitted neutrals lie on a surface of revolution that satisfies the equations of a hyperboloid of one sheet,

$$
\begin{aligned}
x(\gamma, \tau) & =\frac{v \sqrt{1-p^{2}}}{\omega_{c}}[\cos (\gamma)-\tau \sin (\gamma)] \\
y(\gamma, \tau) & =\frac{v \sqrt{1-p^{2}}}{\omega_{c}}[\sin (\gamma)+\tau \cos (\gamma)] \\
z(\gamma, \tau) & =\frac{p v}{\omega_{c}} \tau,
\end{aligned}
$$

where $v$ is the speed of the neutral particle, $\omega_{c}$ is the ion cyclotron frequency, $\tau$ parameterizes the distance along the sheet, and the $\mathrm{z}$ axis is aligned with the magnetic field. The code finds the intersection points of the edges of the aperture and of the detector with this surface, then solves Eq. 8 to find the range of gyroangles, $\Delta \gamma$, that both hit the detector and pass through the aperture. The initial population flux is given by the $n$-level weighted charge-exchange reaction rate of the accepted fast ions with the neutral population. Since the path length of the trajectories within the gyrorange are similar, escaping neutral attenuation is only calculated by the collisional radiative model for the central trajectory. The neutral flux is then equally distributed among the other trajectories in the range. This is done so that the particles that hit the detector are not biased towards the center of the detector. This approach is equivalent with the previously discussed method. Fig. 4 compares the solid-angle approach with the gyroangle approach. Both methods give the same neutral flux.

Since passive NPA calculations require a relatively large spatial grid, passive NPA calculations use the Monte Carlo gyro-angle method, as it is more efficient than the solid-angle approach for spatially extended calculations.

\subsection{Other output}

In addition to active NPA and Balmer-alpha spectra, FIDASIM output now includes many other useful quantities as described in the following.

\subsubsection{FIDA weight functions}

Velocity-space weight functions display the portion of velocity space that is accessed by a given fast-ion diagnostic, for example, the sensitivity of a FIDA channel in a defined wavelength range. In other words, FIDA weight functions show the probability that a fast ion in a certain energy and pitch range will be neutralized and subsequently emit Balmer- $\alpha$ 

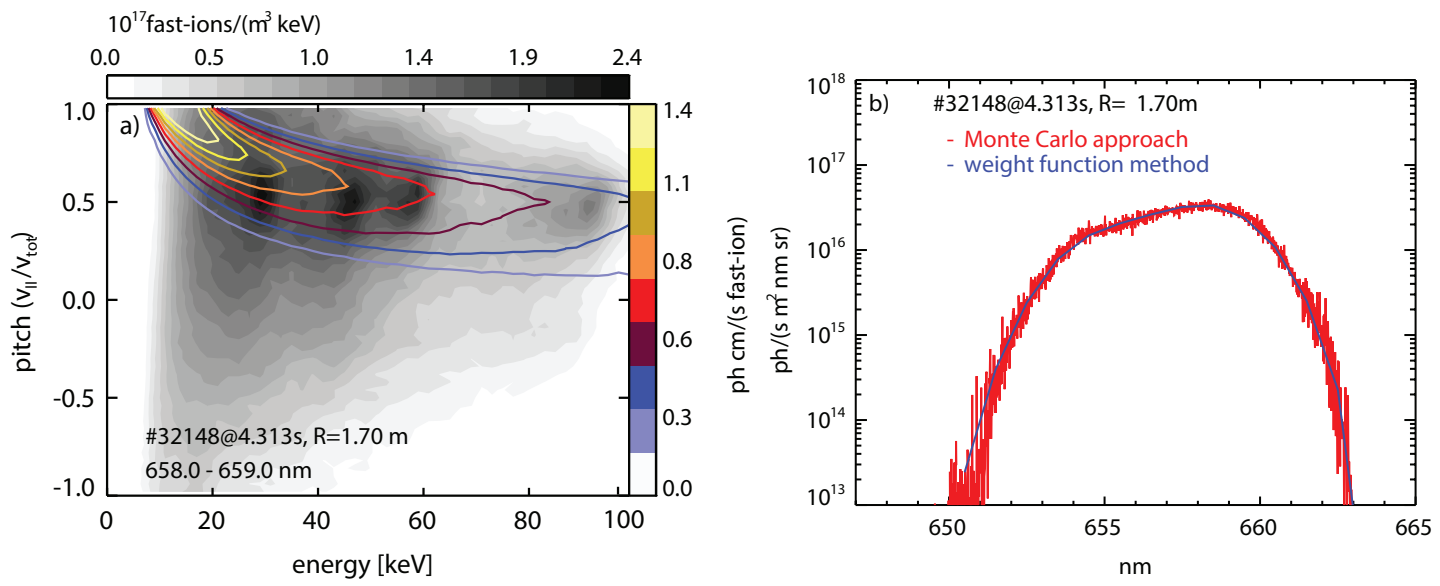

Figure 5: (a) Velocity-space weight function of a toroidal LOS in ASDEX Upgrade for wavelengths between $659.0-659.1 \mathrm{~nm}$. The grey contours show a theoretical fast-ion velocityspace distribution calculated by TRANSP NUBEAM. (b) Simulated FIDA spectra using the Monte Carlo approach (red) and using the weight function method (blue) for the toroidal LOS and distribution function of panel a.

radiation of a given wavelength. The shape of a FIDA weight function in velocity space mainly depends on the geometry of the optical LOS and on the magnetic field configuration $[12]$.

The most general definition of a weight function $W$ is

$$
S=\int W(X) F(X) d X
$$

where $S$ is the diagnostic signal, $F$ is the fast-ion distribution function, and $X$ are the chosen phase-space coordinates. The most commonly used and provided by FIDASIM are velocity-space weight functions $F(E, p)$, where $E$ and $p$ are the fast-ion energy and pitch [13]. In addition, more complete orbit weight functions have e.g. been introduced by [14].

Weight functions have several applications. When signals from different fast-ion diagnostics or lines of sight evolve differently, knowledge of their differing velocity-space sensitivities facilitates interpretation. In addition the weight functions have been used for tomographic inversions of data from multiple FIDA views to infer the velocity-space distribution function $[15,16,17,18,13]$.

The weight functions depend not only on the geometry of the LOS but also on the kinetic plasma profiles, on the magnetic field vectors, and on the density of neutrals. They thus need to be calculated separately for each discharge and time point. 
The calculation of LOS weight functions is based on the assumption that the FIDA radiation is well localized; hence, it is only useful for active FIDA measurements. The FIDA radiation is assumed to originate from the position where the beam and halo density is present which allows the application of line-averaged plasma parameters (weighted means), $\mathrm{X}_{\text {mean }}$, present along a given LOS:

$$
\mathrm{X}_{\text {mean }}=\frac{\int \mathrm{X} \cdot\left(d_{\text {beam }}+d_{\text {halo }}\right) d l}{\int\left(d_{\text {beam }}+d_{\text {halo }}\right) d l}
$$

Here $\mathrm{X}$ represents the various plasmas parameters such as $T_{e}, T_{i}, n_{e}$ or $\mathbf{B}$. $d l$ is along a given LOS and $d_{\text {beam }}$ and $d_{\text {halo }}$ are the density of beam and halo neutrals.

With the knowledge of the line-averaged quantities, the FIDA emission of representative fast-ion velocity vectors can be calculated. Typically, velocity vectors are used that span the relevant fast-ion velocity space with 50 different energies, 50 pitches and 200 gyro angles. For every velocity vector, first, the neutralization rate, i.e. the charge-exchange probability, is calculated using the LOS integrated density of beam and halo neutrals instead of a cellrelated density. In addition, the time, $d t$, needed to pass through the density of beam and halo neutrals is calculated. For this purpose, FIDASIM considers the intersection length with the density of beam and halo neutrals at half maximum.

The charge-exchange probability defines an initial neutral flux that is then applied in the collisional radiative model in order to obtain the quantity of fast neutrals present along the LOS during the time interval $d t$. Here, the line-averaged kinetic plasma parameters are applied. Finally, the spectrum of emitted FIDA light is calculated. The code then checks how much of the predicted spectrum of a velocity vector (fast particle) overlaps with the predefined wavelength range $\left(\lambda_{1}-\lambda_{2}\right)$ of the desired weight function. If the emission falls within the wavelength range, the corresponding photon flux is added to the velocity space bin that describes this fast ion. Fig. 5a shows an example.

As a check on the calculation of the weight function, Fig. 5b compares theoretical FIDA spectra obtained by integrating $W(E, p) F(E, p)$ over velocity space from 14 weight functions (14 wavelengths between $650 \mathrm{~nm}$ and $664 \mathrm{~nm}$ ) with spectra calculated by the standard forward-modeling Monte Carlo approach. The two methods of determining the spectra agree well.

\subsubsection{Radial resolution plots}

Radial resolution plots obtained by storing the neutralization position of fast ions that contribute to a given detector LOS are now available. These plots allow detailed studies of the resolution of FIDA measurements which are affected by electron and ion-impact excitation and subsequent photon emission along the path of a given neutral through the plasma [17]. 


\subsubsection{Bremsstrahlung}

FIDASIM has been equipped with a module to calculate bremsstrahlung emission. The latter is an unavoidable background signal in FIDA spectra and is, for instance, helpful to check the absolute intensity calibration of a FIDA system. The local bremsstrahlung per unit wavelength is calculated using [19]

$$
\frac{d N_{B}}{d \lambda}=7.57 \times 10^{-9} g \frac{n_{e}^{2} Z_{e f f}}{\lambda T_{e}^{1 / 2}} e^{-h c / \lambda T_{e}},
$$

where $\lambda$ is the wavelength in angstroms, $n_{e}$ and $T_{e}$ is the electron density in $\mathrm{cm}^{-3}$ and temperature in eV respectively. The gaunt factor, $g$, depends on $T_{e}$ and $Z_{\text {eff }}$. It can be approximated by [19]

$$
g=5.542-\left(3.108-\ln \left(T_{e} / 1000\right)\right)\left(0.6905-0.1323 / Z_{\text {eff }}\right) .
$$

To calculate the total emission, the local emissivity is integrated over the line of sight.

\subsubsection{Neutron rates}

The expected volume-averaged beam-target d-d neutron rate can be calculated which allows one to check the validity of the input data, in case neutron measurements are available. Details on this calculation are given in Sec. 3.6 of [20] and the calculation was verified through comparison with TRANSP.

\subsubsection{Passive Simulations - Cold neutrals}

The simulation of passive FIDA and NPA fluxes has gained increasing attention in recent years since it turned out that FIDA and NPA signals can be significantly affected by passive contributions $[21,22]$. In addition, charge exchange of fast ions has been identified as an significant loss mechanism for instance at the TCV tokamak and the corresponding passive FIDA signal provides a direct measure to probe these losses [23]. FIDASIM has therefore been upgraded to be additionally capable of modelling passive NPA and FIDA signals; active and passive signals do now appear as separate output in the code. The simulation of passive signals requires a 1D or 2D background neutral distribution as input. Typically the output from TRANSP is used here, which provides 1D neutral density profiles as calculated by the FRANTIC sub-module. Since these neutral density profiles are energylevel integrated, FIDASIM uses its collisional radiative model to obtain $n$-resolved neutral densities: based on the local plasma parameters, FIDASIM determines the equilibrium distribution of hydrogenic atoms assuming that the neutral temperature is equal to the local ion temperature. The resulting neutral population is then used to calculate charge exchange probabilities with ions from a given distribution function. From this point on, the resulting neutralized fast ions (or thermal ions) are treated similarly to those arising 
from charge exchange reactions with beam and halo neutrals. An comparison of predicted and simulated passive FIDA spectra will be shown in Fig. 11, as a part of the validation section 3 .

\subsubsection{Fast-ion birth profiles}

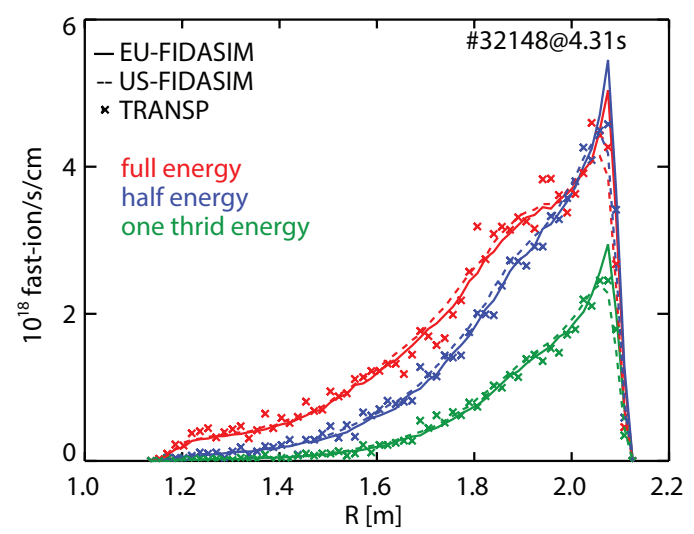

Figure 6: Birth profile of fast ions with full, half and one third of the injection energy $(60 \mathrm{keV})$ as calculated by the EU- and US-versions of FIDASIM. The crosses are the results from TRANSP.

FIDASIM has been equipped with the ability to provide fast-ion birth profiles. The calculation is similar to the determination of the neutral beam density. However, the flux of ionized neutrals and corresponding velocity vectors are stored per grid cell rather than the density of neutrals. Example profiles from FIDASIM are shown in Fig. 6 for the full, half and one third energy component. The profiles are compared with data from TRANSP which agrees very well in shape and magnitude. The birth profiles from FIDASIM have e.g. been coupled with a solution of the Fokker-Planck equation to prototype codes which calculate the NBI fast-ion distribution faster than TRANSP [24]. In addition, FIDASIM is capable of calculating birth profiles even outside the last-close flux surface which can be used for instance in full-orbit simulations to determine prompt-losses.

\section{Code verification and validation}

Because significant changes were made to the code compared to the original IDL version of FIDASIM, verification and validation is crucial. The simulation of the FIDA spectra has already been verified, as two different methods - the standard Monte Carlo approach (appendix. E) and the approach using weight functions (Sec. 2.4.1) - give very similar results (Fig. 5). In addition, the good agreement between radial birth profiles from TRANSP with results from FIDASIM (actually from the EU and US version) in Fig. 6 suggests that the 
calculation of the attenuation of neutrals, is consistent. However, these comparisons do not fully validate the new collisional radiative model as this requires for instance a n-level resolved analysis rather than the ionization profile. Moreover, the method of calculating the halo-distribution needs to be verified as this is one of the main improvements compared to the original version of FIDASIM.

Hence, this section presents comparisons of the collisional-radiative model with the Atomic Data and Analysis (ADAS) compilation [25], calculations of the NBI footprint with a measured image, and the simulated beam, halo and FIDA spectra with measurements. Finally, the impact of large fast-ion populations on the FIDASIM results is discussed.

\subsection{Collisional-radiative model}
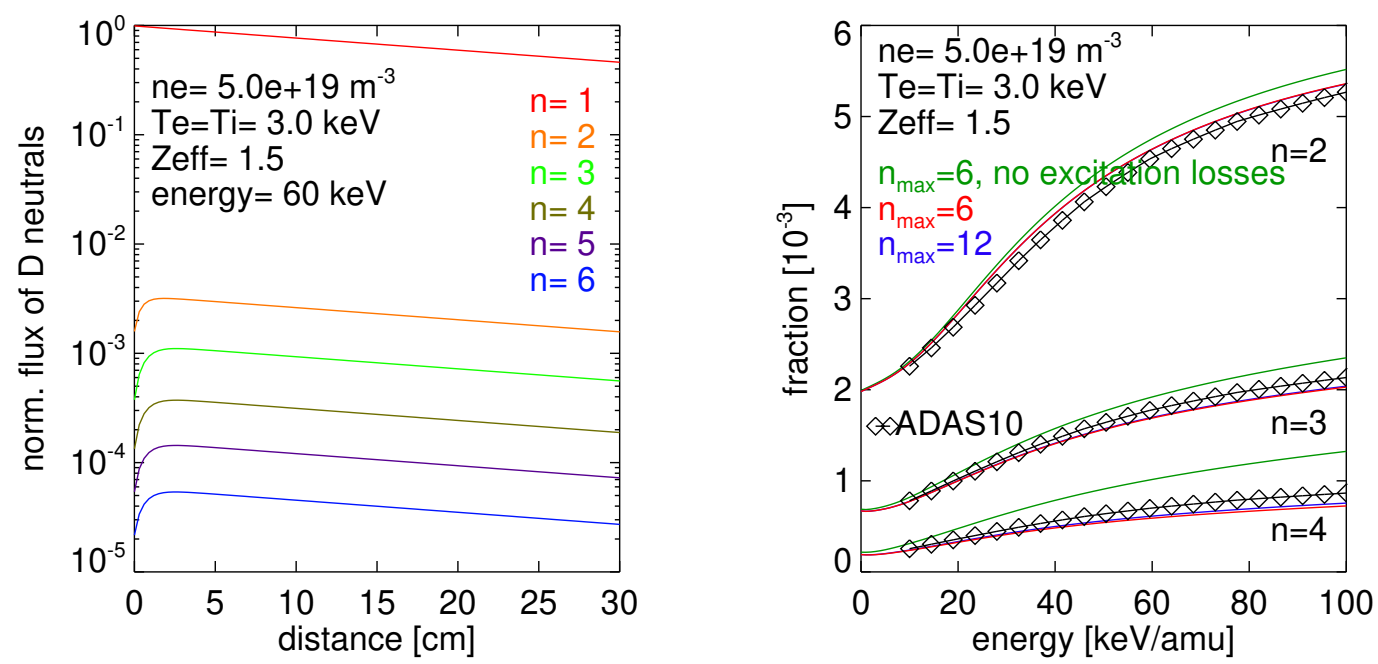

Figure 7: a) Flux of $60 \mathrm{keV}$ neutrals on a semi-logarithmic scale that are initially in the ground state. b) Relative equilibrium populations of the $n=2-4$ states for neutrals with energies between 0 and $100 \mathrm{keV} / \mathrm{amu}$ when using 6 (red) and 12 (blue) energy levels. In black, the relative populations determined by the collisional radiative model of ADAS are plotted. The green curves show the erroneous values that occur when impact excitation of high-energy states is neglected in a simulation that uses levels $n=1-6$.

Fig. 7 shows results of the FIDASIM solution of the collisional radiative model. In Fig. 7a, the evolution of the flux of $60 \mathrm{keV}$ deuterium neutrals that are initially in the ground state is shown. Within the first few centimeters along their path, the excited states are populated, and an equilibrium population is reached after about $2 \mathrm{~cm}$. The population of the excited states is orders of magnitudes lower than the population of the ground state $(n=1)$. With 
increasing distance, more and more neutrals become ionized which reduces the total flux of neutrals. In Fig. 7b, the relative steady-state populations of the $n=2, n=3$ and $n=4$ states are plotted as a function of energy. In blue, the resulting fractions are shown when considering 12 excited states in the collisional radiative model of FIDASIM rather than the standard 6 . The result is very similar to the one when assuming only 6 excited states. However, if impact excitation into $n>6$ levels is neglected and not considered a loss mechanism, the discrepancy with respect to the ADAS values becomes significant (as shown in green). In black, the relative population of the $n=2-4$ states determined by ADAS [25] routines are shown. Good agreement is obtained with the collisional radiative model in FIDASIM, which proves the reliability of the implemented cross sections and routines.

\subsection{Validation of the halo model by comparisons with TRANSP}

In a previous publication [26], FIDASIM output was compared with output from the TRANSP code for a representative NSTX case. Good agreement was reported for synthetic NPA measurements and for the injected neutral profile. In addition, the shape of the 3D halo profile agreed well as the FIDASIM multi-halo-generation scheme had been implemented in TRANSP but the halo density differed by $\sim 15 \%$. This difference was attributed to differences in atomic cross sections in FIDASIM and TRANSP.

\subsection{Validation using a beam imaging diagnostic}

An ASDEX Upgrade beam imaging diagnostic has been used to verify the description of the NBI geometry used in FIDASIM and to verify the simulation of beam and halo neutrals. The beam imaging diagnostic has a viewing geometry similar to the toroidal view of the FIDA diagnostic and observes radiation in the visible range with a CMOS camera. The camera operates with a time resolution of $200 \mu$ s and uses $256 \times 256$ pixels. In low density plasmas, a clear contribution from the beam and halo radiation is visible in the camera frames. Fig. 8a shows a camera frame that has been measured in ASDEX Upgrade discharge \#26381. In Fig. 8b, the same camera frame is shown in which the background radiation, observed when NBI was off, has been subtracted. The active radiation observed by the camera mainly consists of D-alpha radiation from beam and halo neutrals, as the active radiation from other contributions such as impurity charge exchange lines are much weaker in the visible range. Therefore, the measured footprint can be compared with the beam and halo radiation predicted by FIDASIM.

The beam and halo radiation has been calculated by FIDASIM for 4096 LOS that correspond to the viewing directions along every 16th camera pixel. The sum of the resulting halo and beam spectra have been integrated in wavelength for every LOS and are shown in Fig. $8 \mathrm{~b}$ by red contour lines. To be able to better compare the simulation to the measurement, Fig. 8c,d shows the data from vertical and horizontal cuts through the cam- 

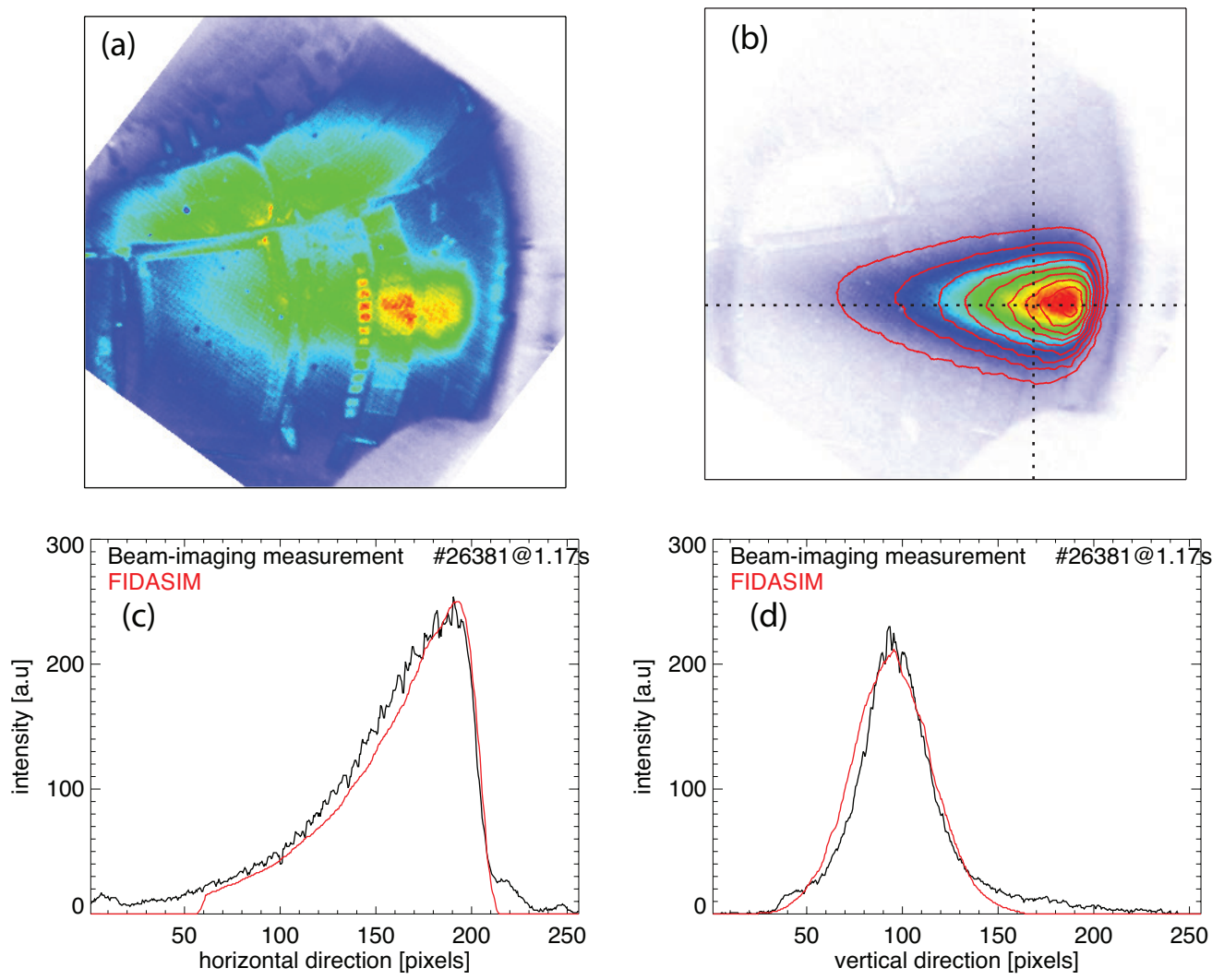

Figure 8: (a) Total light measured by an ASDEX Upgrade beam-imaging diagnostic during NBI and (b) with background frame subtracted. The simulation from FIDASIM is shown with red contour lines. (c) Horizontal and (d) vertical cuts through the active image along the dotted lines shown in panel $b$.

era image. As can be seen, excellent agreement is obtained between the measurement and the simulation. This indicates that the geometry of the NBI in terms of the vertical angle, focus and divergence is well described by the inputs to FIDASIM. Moreover, the good match between the predicted attenuation of NBI and the measurement shows that the collisional radiative model used in FIDASIM is valid. Lastly, it is worth mentioning that the wavelength-integrated intensity of the halo emission intensity is similar to that of the beam emission. The good match between the measured and simulated footprint of NBI consequently shows that the model of the halo contribution used by FIDASIM is appropriate. 

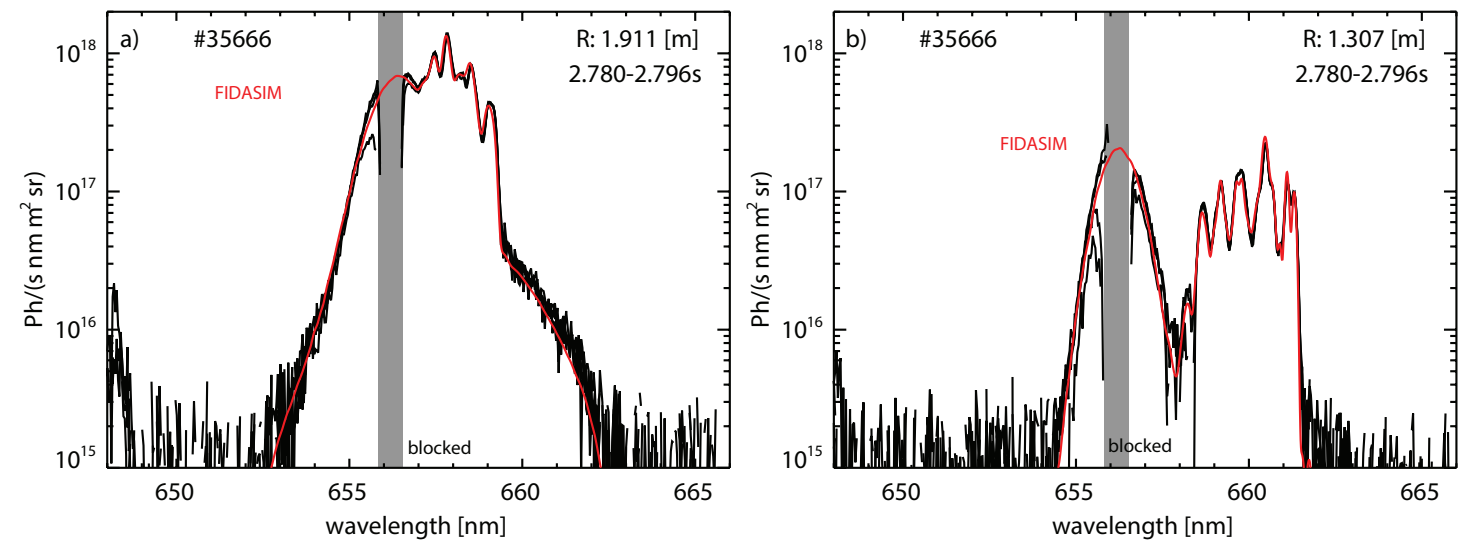

Figure 9: Measured active spectra of an ASDEX Upgrade beam-emission diagnostic at two radial positions and four successive time points. At $656.1 \mathrm{~nm}$, the intense cold, passive $\mathrm{D}_{\alpha}$ radiation from the plasma edge is present which is blocked by a wire inside the spectrometer setup. The sum of the simulated beam-emission, halo and FIDA contributions is displayed in red and fits the measurement well in shape and intensity.

\subsection{Validation using Balmer alpha spectroscopy diagnostics}

Measurements from an absolutely calibrated beam emission spectroscopy diagnostic at ASDEX Upgrade have been used to check and validate FIDASIM. The diagnostic is based on an array of toroidal LOS that intersect a $93 \mathrm{keV}$ NBI source at different radial positions from the low-field-side (LFS) to high-field-side (HFS) of ASDEX Upgrade [27]. A spectrometer with high spectral resolution and a wire to block the un-shifted cold D-alpha emission is used that allows measuring the red and the blue shifted D-alpha radiation without saturation effects on the CCD camera attached to the spectrometer exit. In Fig. 9a and $b$, active spectra (the passive radiation without NBI has been subtracted) from a LFS-LOS $(\mathrm{R}=1.9 \mathrm{~m})$ and a HFS-LOS $(1.3 \mathrm{~m})$ are shown, which were acquired during an MHD-quiescent plasma experiment (\#35666). The beam emission can be observed at redshifted wavelengths (roughly between $657 \mathrm{~nm}$ and $662 \mathrm{~nm}$ ) with larger Doppler shifts at the high-field-side $(1.3 \mathrm{~m})$, explained by a less perpendicular angle between the NBI and the LOS. Due to the beam-attenuation, the intensity of the beam emission is reduced for the HFS-LOS (note the semi-logarithmic scale) and a stronger Stark-splitting is observed since the magnetic field strength is notably larger on the HFS. These features are well recovered by the synthetic spectra from FIDASIM, plotted in red. The shape, as well as the intensity, of the measured beam emission agrees very well with the simulation. This demonstrates that parameters such as the species mix, the geometry, the divergence, the power, and the injection energy are well described by FIDASIM. Furthermore, the good agreement shows that the input kinetic plasma profiles, as well as the applied collisional radiative model, 
are appropriate. In addition, the simulated spectra contain the halo-emission which has a Gaussian shape close to $656.1 \mathrm{~nm}$. The good agreement between measurement and simulation indicates that the new approach - describing the halo neutrals by several generations - is valid. Finally, it is also worth mentioning that the FIDA emission (the broad feature visible in Fig. 9a) describes the data very well. The FIDA emission has been calculated based on a neo-classical fast-ion distribution function from TRANSP which is expected to describe MHD-quiescent plasma conditions reasonably well.
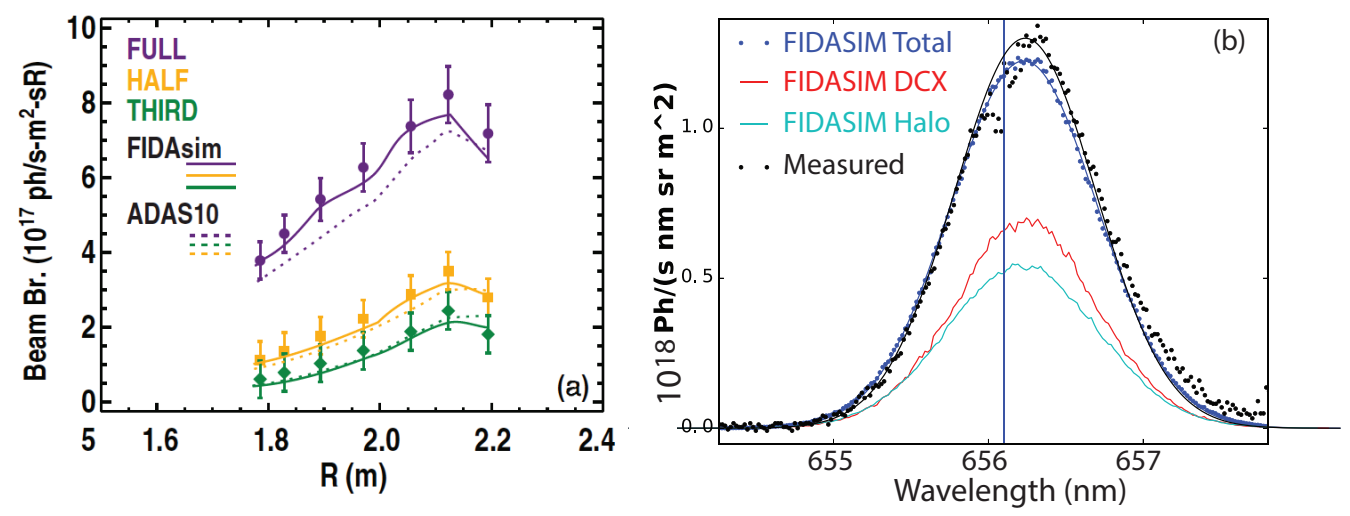

Figure 10: Validation by the DIII-D main-ion charge exchange recombination diagnostic. (a) Measured radial profiles (points with error bars) of full-energy, half-energy, and thirdenergy beam emission from an $80.8 \mathrm{keV}$ deuterium beam in a discharge with $\bar{n}_{e} \simeq 2.6 \times$ $10^{19} \mathrm{~m}^{-3}$. The profiles predicted by FIDASIM (solid lines) and by a calculation using rates from ADAS 10 (dashed lines) are also shown. Reproduced with permission from [28]. (b) Active spectrum from thermal deuterium ions (black points) and FIDASIM predictions (blue points) in an H-mode discharge at a major radius of $218.6 \mathrm{~cm}$. The FIDASIM prediction is the sum of DCX light produced in the first charge-exchange generation (red line) and the halo light produced in subsequent generations (blue line). The vertical line is the un-shifted rest $\mathrm{D}_{\alpha}$ wavelength. Reproduced with permission from [29].

Moreover, measurements by the absolutely calibrated main-ion charge exchange recombination diagnostic at DIII-D [28] compare well with FIDASIM calculations. This diagnostic employs tangential views of modulated heating beams in the midplane. Several examples of successful comparisons were previously published [28, 29]. Fig. 10(a) shows that the radial profile of the beam emission agrees well with FIDASIM predictions for all three energy components of the injected beam. An example of prediction and interpretation of the spectrum produced by thermal deuterium is shown in Fig. 10(b). As Ref. [29] stresses, FIDASIM's full collisional-radiative model is particularly valuable in regions with steep gradients in plasma parameters, such as the pedestal at the edge of a tokamak H-mode.

A direct comparison between measured and predicted passive FIDA signals has e.g. been 


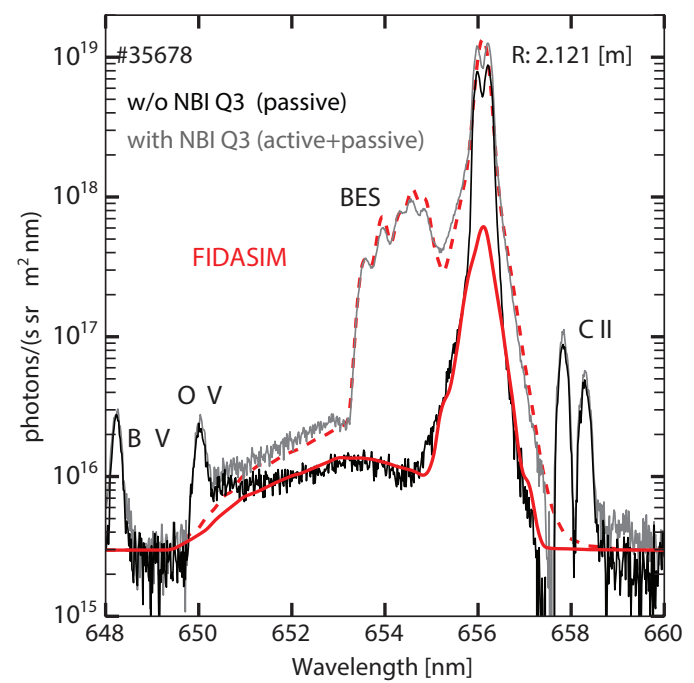

Figure 11: Passive edge FIDA spectra measured at ASDEX Upgrade during 2.5 MW of off-axis NBI heating (black), together with active+passive spectra during a beam blip from NBI Q3 (gray). The predicted passive FIDA contribution from FIDASIM is plotted by a solid red line. Note here, that the thermal ion distribution function has additionally been considered in the passive simulation. The sum of simulated active + passive contributions is illustrated by a red, dashed line and agrees well with the measurements. Reproduced with permission from [21].

published by [21]: A dedicated edge-FIDA diagnostic system has been installed at ASDEX Upgrade which observes particularly strong passive FIDA signals during off-axis NBI injection. Fig. 11 shows a passive measurement during $2.5 \mathrm{MW}$ of off-axis NBI heating in a low-density H-mode plasma. A strong passive FIDA contribution can be observed which is reproduced reasonably well by FIDASIM using a TRANSP-predicted fast-ion distribution function and a 1D neutral density profile from KN1D [30]. In addition, a thermal distribution function has been considered in FIDASIM which allows modelling the edge Dalpha radiation after charge-exchange reactions between thermal ions and neutrals. This thermal component agrees well with the wings of the thermal passive emission. Only the passive D-alpha radiation from cold incoming (recycling) neutrals is not considered such that measurement and simulation differ close to $656 \mathrm{~nm}$. In addition to the passive radiation, an active+passive spectrum was obtained during a short blip of a neutral beam source (labelled Q3) which serves as a diagnostic beam and demonstrates the applicability of FIDASIM also for edge-localized measurements. 

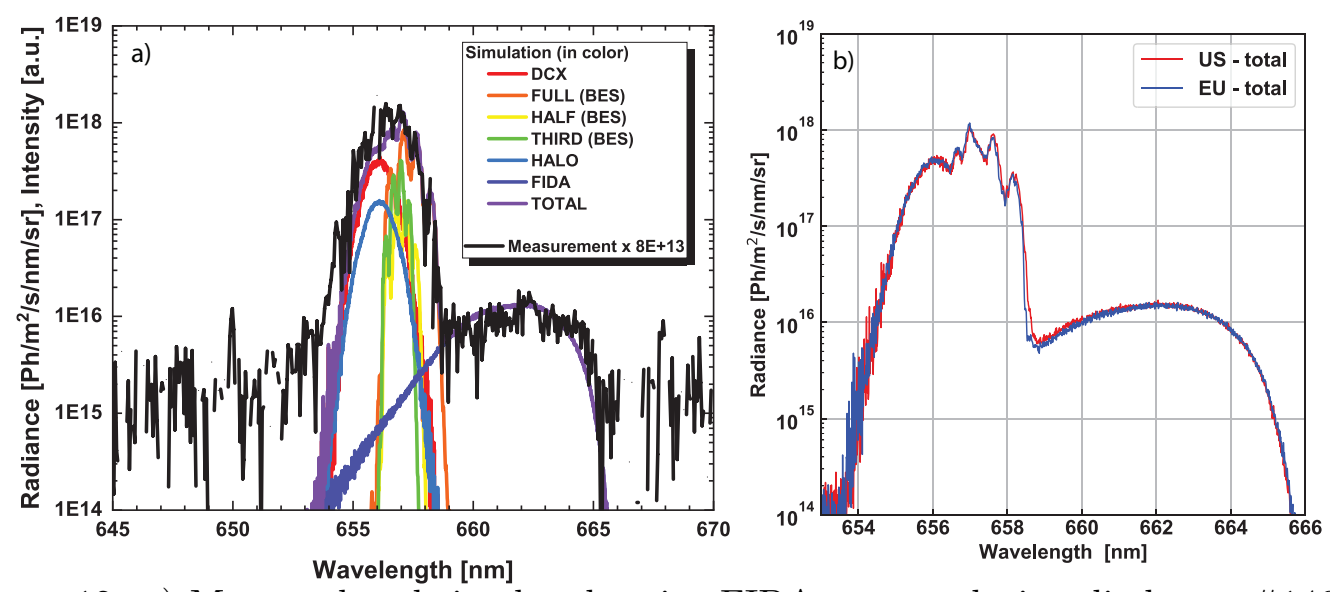

Figure 12: a) Measured and simulated active FIDA spectra during discharge \#146695 at LHD. The shapes of the predicted beam emission, of the DCX and halo light, and of the FIDA radiation agree well with the measurement. b) Comparison between the predicted FIDA spectra using the US and EU versions of FIDASIM as discussed in section 4.

\subsection{Validation of the three-dimensional capability}

The capability to model three-dimensional plasmas has been tested in a study at the Large Helical Device (LHD [31]) using a $174 \mathrm{keV}$ co-injected deuterium negative neutral beam with $0.7 \mathrm{MW}$ of power. For active FIDA spectroscopy, an array of tangential lines of sight is used that intersects a positive deuterium neutral beam [32] that is on-off modulated. Fig. 12a shows an active spectrum from a central line of sight that was obtained during an MHD-quiescent experiment with a line-average electron density of $\sim 0.65 \times 10^{19} \mathrm{~m}^{-3}$ and additional $2.7 \mathrm{MW}$ of electron cyclotron resonance heating. In addition to the experimental data in black, predictions from FIDASIM are plotted in color. Note here that the measured spectrum is not absolutely calibrated and has been scaled to match the simulation. Moreover, the data in black features a relatively strong noise level which yields discontinuous lines in the wings of the spectrum; the data can be negative or positive but the semi-logarithmic plot only shows positive values. However, the observed large Doppler shifts of the FIDA radiation, as well as the overall shape of the Balmer alpha spectrum agree well with the simulation. This demonstrates that FIDASIM is a valuable tool also for 3D magnetic field configurations. More details on the application of FIDASIM at LHD will appear in a forthcoming publication [33].

\subsection{Simulations in presence of large fast-ion populations}

The collisional radiative model of FIDASIM does typically not consider fast ions. Instead, the tabulated charge exchange, impact ionization and (de-)excitation reaction rates assume a Maxwell distribution and are multiplied with the total deuterium ion density (thermal 

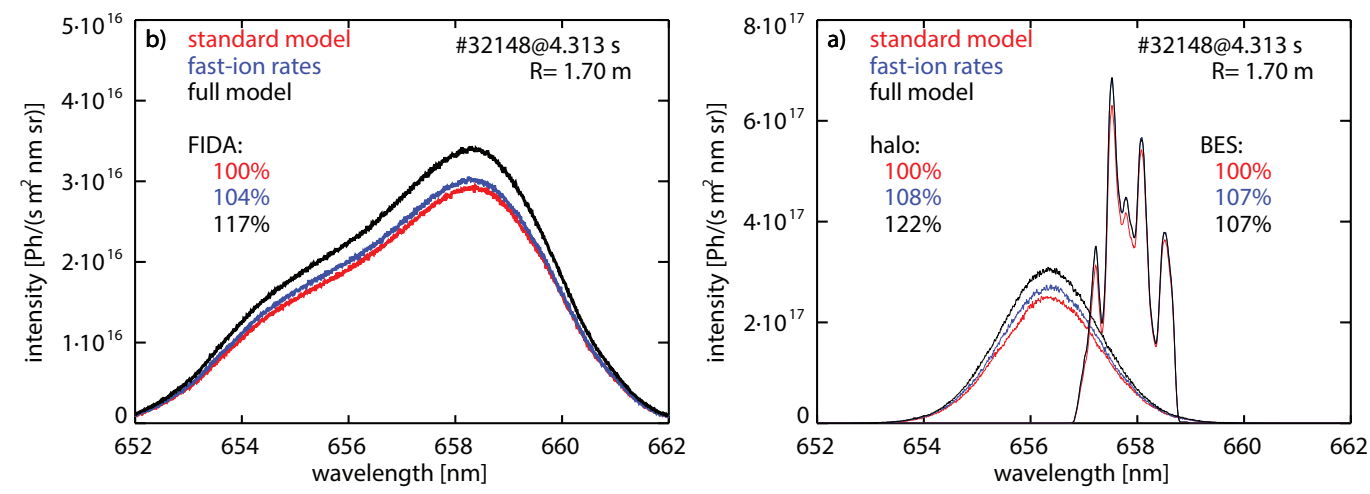

Figure 13: Predicted beam emission and halo spectra (a), as well as FIDA spectra (b), for AUG discharge \#32148. The simulation in red represents the standard approach in FIDASIM, while the simulation results in blue take reaction rates, modified by the fastion distribution function into account (labelled 'fast-ion rates'). Finally, the synthetic spectra in black additionally consider the halo formation via charge exchange reaction of fast neutrals (labelled 'full model').

ions + fast ions). However, in the presence of large fast-ion densities, the actual reaction rates might differ. FIDASIM has therefore been equipped with the option to perform computationally more expensive simulations that consider the presence of fast particles. During these simulations, the reaction rates of the thermal plasma are taken from the tabulated values as before. However, collisions with the fast-ion population are treated separately by calculating reaction rates between a given neutral Monte Carlo marker and one randomly selected fast-ion velocity vector whenever the collisional radiative model is called. Since the collisional radiative model is called millions of times during FIDASIM simulations, considering only one fast-ion velocity vector is sufficient to maintain good statistics. Fig. 13 compares predicted FIDA spectra of the representative AUG experiment \#32148 already introduced in section 2.2. This experiment featured a central fast-ion fraction of $30 \%$ (relative to the total deuterium ion density) and a clear effect on the predicted FIDA spectrum is seen. The simulation data in blue (fast-ion rates) accounts for the modified reaction rates and has a stronger beam emission, halo emission and FIDA emission than the simulation in red representing the standard case.

In addition, Fig. 13 shows in black simulation results for which the effect of thermal ions getting neutralized by charge exchange reactions with fast neutrals was considered additionally to the fast-ion rates. This "fast halo" effect can be accounted for by storing the fast-neutral distribution function on the FIDASIM simulation grid. Then, after a first iteration of FIDASIM, a second iteration can be started during which the stored fast neutrals are used the same way as the stored density of beam neutrals was applied during the first iteration: Based on the fast-neutral distribution function, the charge exchange probabil- 
ity for reactions with thermal ions is determined and new DCX and halo simulations are started. This new halo population adds to the total halo population and can be used to calculate the additional FIDA radiation. The impact on the halo emission of this second iteration is about $14 \%$ for the representative AUG case shown in Fig. 13. Note here that a third iteration would only modify the result by about $1 \%$ and has therefore been ignored. The difference between the standard simulation and the more complete (full) model demonstrates that in presence of large fast-ion densities, results from standard FIDASIM simulations need to be evaluated with care.

\section{Benchmark between EU and US versions of FIDASIM}
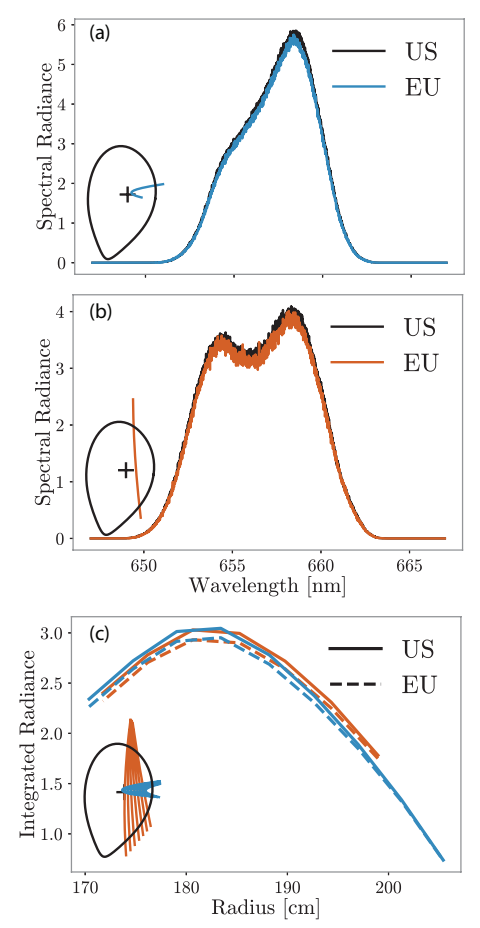

Figure 14: Comparison of USA and EU calculations of the active FIDA emission for an ASDEX-Upgrade H-mode plasma during on-axis neutral beam injection (\#32148). Spectra for (a) a tangential LOS and (b) a poloidal LOS; spectral radiance is plotted in units of $10^{16}$ photons/(s-nm- ${ }^{2}$-sr). (c) Integrated radiance vs. major radius for the tangential (blue) and poloidal (red) arrays in units of $10^{17}$ photons $/\left(\mathrm{s}-\mathrm{m}^{2}-\mathrm{sr}\right)$. The diagrams in the lower left corners show LOS projections onto the plasma cross section.

As mentioned in the introduction, after completion of the initial conversion of FIDASIM from IDL to FORTRAN in 2013 [34], separate versions with additional functionality were 
developed in both Europe and the USA. Main differences are an adaptive step-size when following Monte Carlo markers in the EU-FIDASIM version, different schemes interpolating kinetic profiles, magnetic fields and the fast-ion distribution function and the use of different parallelization tools; MPI is typically used by the US version and openMP by the EU one. In 2018, we decided to reunify the codes and embarked on a set of benchmark activities. The selected comparison cases vary greatly in plasma conditions.

- A high-density ASDEX-Upgrade H-mode plasma (\#32148).

- A low-density L-mode plasma from the DIII-D tokamak with a high fast-ion fraction.

- A low field NSTX plasma where gyroradius effects are important.

- A Tokamak à Configuration Variable (TCV) plasma with large passive signals.

- A low density LHD stellarator plasma with fast ions from a negative neutral beam.

These comparison activities revealed some discrepancies that resulted in bug-fixes on both sides. The two versions now agree well.

The two versions of FIDASIM are compared in Fig. 14 for the representative ASDEX Upgrade H-mode deuterium plasma already introduced earlier. This experiment featured two phases. First, pure on-axis NBI heating was applied until $4.5 \mathrm{~s}$. Then, two $93 \mathrm{keV}$ on-axis beams were replaced by two $93 \mathrm{keV}$ tangential off-axis beams (as in a previous experiment [16]), while the $60 \mathrm{keV}$ on-axis beam needed for FIDA spectroscopy ran throughout the entire discharge. The results from the two codes agree to within $3 \%$, i.e. USA $\approx 1.03 \times \mathrm{EU}$, for both on-axis and off-axis NBI. This is not immediately expected given the many differences between the two codes but the excellent agreement shows that all relevant processes are correctly modelled in both codes.

Fig. 15 compares D-alpha spectra for the DIII-D L-mode. The qualitative agreement is excellent; the quantitative agreement is similar to the ASDEX-Upgrade case.

Examples of good agreement in the NPA calculations between the EU and US versions of FIDASIM appear in Fig. 16 for the active signal in TCV and the passive signal in NSTX.

Owing to the large neutral beam injection energy and low field, an improved treatment of finite Larmor radius effects (see Appendix E) is particularly important in spherical tokamaks such as NSTX. The effect of these changes shows up most clearly in radial profiles for poloidal lines of sight. When using a guiding-center distribution function, FIDASIM normally displaces the Monte Carlo marker by a gyroradius before tracking the neutral. Both the EU and the USA versions can "turn off" this finite Larmor radius (FLR) step. The EU version uses the leading-order approximation for the gyroradius step; the USA version can use either this "v $\times \mathbf{B}$ " approximation or include higher-order terms. Figure 17 compares NSTX radial profiles for five different calculations. Neglect of the gyroradius 


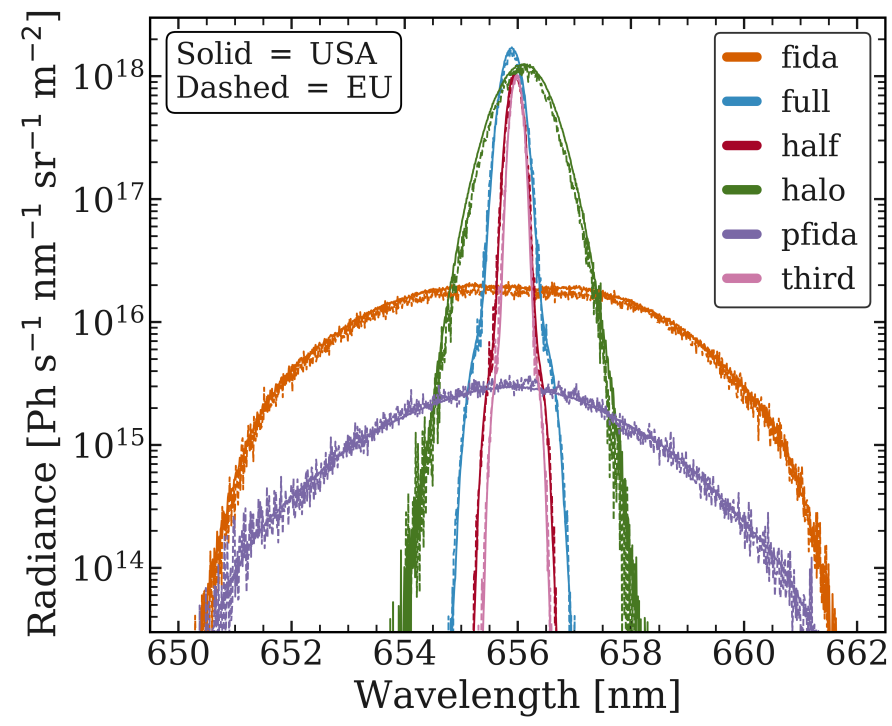

Figure 15: Contributions to the $\mathrm{D}_{\alpha}$ spectrum in a low-density $\left(\bar{n}_{e}=2.1 \times 10^{19} \mathrm{~m}^{-3}\right)$ DIII-D discharge with large fast-ion fraction for a vertical LOS as calculated by the EU (dashed) and USA (lines) versions of FIDASIM. The curves labeled full, half, and third are the beam emission from the $70 \mathrm{keV}$ active beam. The curve labeled halo includes DCX light. The curve labeled "fida" is the active FIDA signal; "pfida" is the passive signal. Although the actual discharge, \#159243 at $430 \mathrm{~ms}$, had strong Alfvén eigenmode activity that caused appreciable fast-ion transport $[35,36]$, the calculation uses the neoclassically predicted fast-ion distribution.

step shifts the profile over $10 \mathrm{~cm}$, a large error. The higher-order terms only shift the profile 1-2 cm compared to the leading-order correction. Comparison of the EU and USA calculations when using the same FLR treatment shows that they are similar in shape but the USA calculation is $\sim 5 \%$ larger and differs by $1-2 \mathrm{~cm}$ in profile shape.

Finally, the 3D capabilities of the US and EU FIDASIM versions have been compared based on the LHD stellarator case already discussed in the validation section 3 . As can be seen in Fig. 12b, excellent agreement in the spectral shape and intensity is obtained between the two codes. This demonstrates that the intrinsic 3D capability of FIDASIM - a 3D simulation grid is used- provides a reliable tool for fast-ion transport studies in stellarators. 

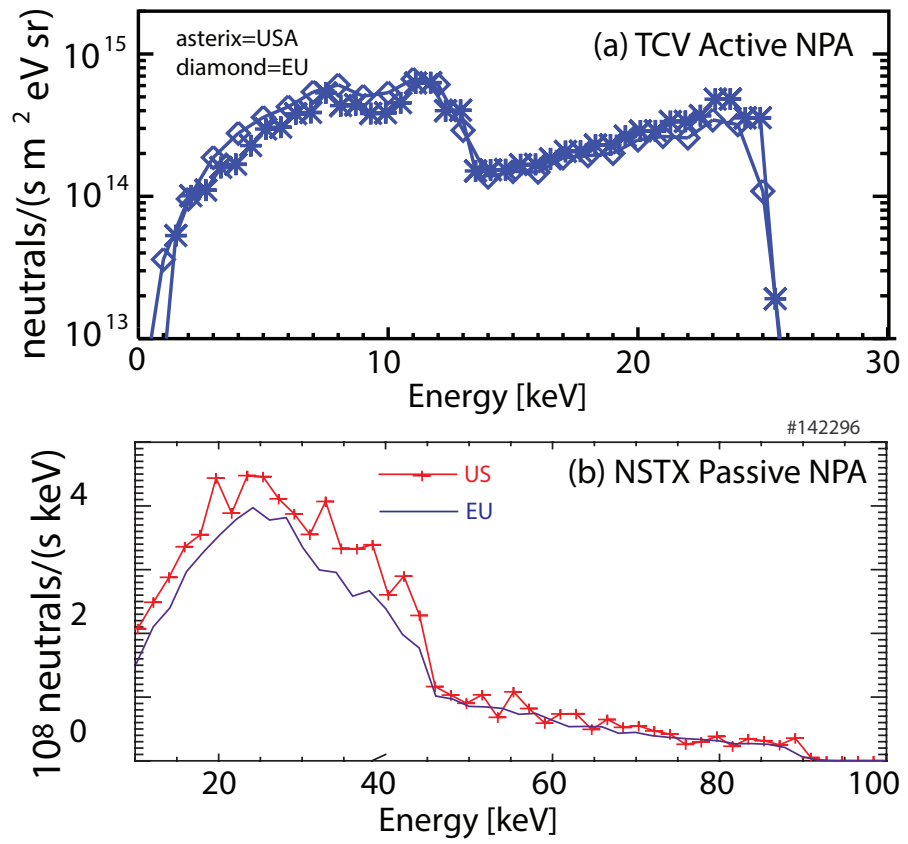

Figure 16: Comparison of NPA spectra computed by the EU and US versions of FIDASIM. (a) Active flux for the tangential LOS of the CNPA diagnostic [37] for TCV plasma \#60923, a plasma with $\sim 0.8 \mathrm{MW}$ of $\sim 24 \mathrm{keV}$ NBI that resembles the discharges described in [38]. (b) Passive flux for a poloidal LOS with tangency radius of $30 \mathrm{~cm}$ for NSTX discharge \#142296, a 0.4 T plasma with $1.9 \mathrm{MW}$ of $90 \mathrm{keV}$ NBI.

\section{Conclusion and Outlook}

FIDASIM is well verified and validated and can be relied upon for accurate calculations of neutral beam deposition, injected and halo neutral densities, Balmer-alpha light from BES, DCX, halo, and active and passive FIDA processes, active and passive NPA emission, and weight functions. Calculations for both axisymmetric and 3D configurations are available.

There are known limitations. The present version of the code only accommodates a single hydrogenic species but work is underway to accommodate multiple species. Although fast neutrals behave differently than thermal neutrals, the present version of the code assumes that halo neutrals originate from reactions with thermal ions; this may affect the results when fast ions are a large fraction of the electron density. In addition, the atomic rates such as ion-impact ionization assume a thermal background plasma. In the case of very large fast-ion densities, this provides additional uncertainties.

Going forward, only a single version of FIDASIM will be maintained, an international 


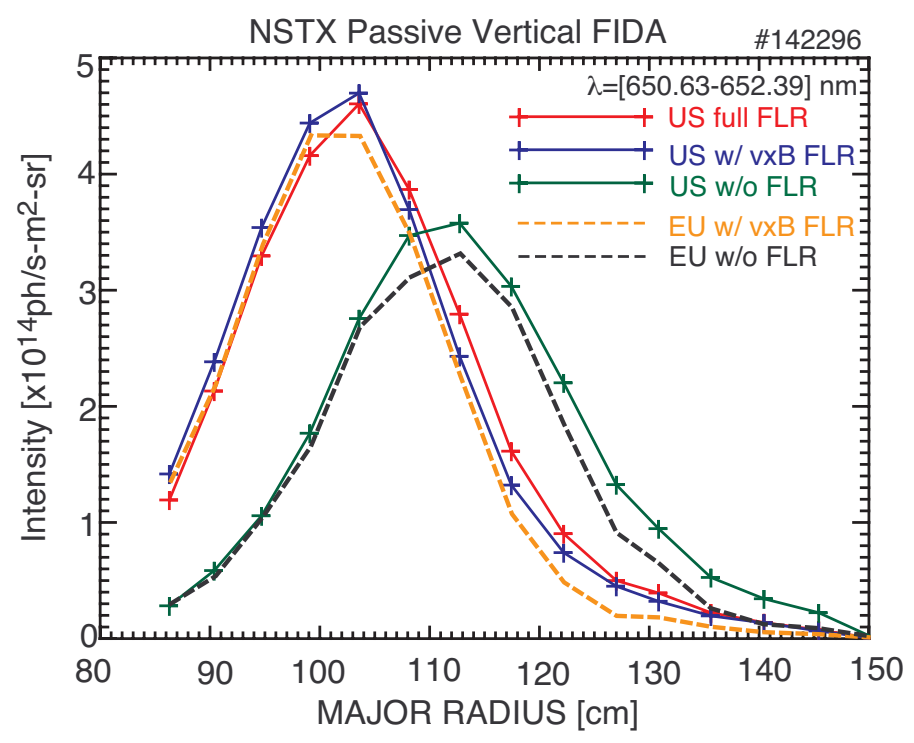

Figure 17: Radial profiles of the wavelength-integrated passive FIDA predictions for the poloidal NSTX array [39] for the US (solid lines, + symbols) and EU (dashed lines) versions of FIDASIM. The five curves are: No finite Larmor radius (FLR) correction for both the EU (black) and USA (green) versions, with leading-order FLR correction for both the EU (yellow) and USA (blue) versions, and with the full FLR treatment (red) for the USA version. NSTX discharge \#142296, a 0.4 T plasma with $90 \mathrm{keV}$ deuterium NBI by source B.

version based on the well documented USA version [8], with additional features of the EU-version incorporated. These features are in particular the use of an adaptive step size when following markers through the $3 \mathrm{D}$ simulation grid, the storage of velocity vectors of neutrals and the on-the-fly atomic rate calculation to consider larger fast-ion populations. Finally we would like to note that new users and developers are welcome.

\section{Acknowledgements}

The support of the ASDEX-Upgrade, DIII-D, LHD, TCV, and NSTX teams is gratefully acknowledged. We also thank the many FIDASIM users who have suggested improvements and identified errors in the code. This work was supported by the U.S. DOE (DE-FC0204ER54698, DE-FG02-06ER54867, DE-SC0019253), by U.S. National Science Foundation 1633631, by the NINS program of Promoting Research by Networking among Institutions (Grant Number 01411702), the NIFS International Collaboration Research programs (NIFS18/KLPR047 and NIFS07/KLPH004), and the LHD project budget (ULRR006, 
ULRR035, ULRR036, and ULRR702). A part of this work was performed on "Plasma Simulator" (FUJITSU FX100) of NIFS with the support and under the auspices of the NIFS Collaboration Research program NIFS18KNST135 and NIFS19KNSR010.

In addition, this work has been carried out within the framework of the EUROfusion Consortium and has received funding from the Euratom research and training programme 2014-2018 and 2019-2020 under grant agreement No 633053. The views and opinions expressed herein do not necessarily reflect those of the European Commission. Moreover, this work was supported in part by the Swiss National Science Foundation. 


\section{APPENDIX}

\section{A Input data}

The code requires profiles of electron and ion temperature $T_{e}$ and $T_{i}$, electron density $n_{e}$, and $Z_{e f f}$, as well as all three components $\left(v_{R}, v_{\phi}, v_{z}\right)$ of the plasma rotation. For a passive simulation, profiles of the cold neutral density are also required. The present version of FIDASIM supports a single fast-ion and active beam species, a single hydrogenic thermal ion species, and a single impurity species. With $n_{e}, Z_{e f f}$, and the fast-ion density known, the densities of the thermal-ion and impurity species are inferred through quasineutrality.

FIDASIM accepts three different ways to provide information on the fast-ion distribution function: A guiding center distribution function, a guiding center Monte Carlo particle distribution, or a full-orbit Monte Carlo particle distribution. For the guiding center distributions, the velocity coordinates are the fast-ion energy $E$ and the pitch (relative to the magnetic field) $v_{\|} / v$. For the full-orbit Monte Carlo distribution, all three velocity components are specified. Spatially, guiding center distribution functions are provided in cylindrical coordinates and are mapped onto the cylindrical interpolation grid; for Monte Carlo distributions, the particle position is a direct input for each marker. Additionally, Monte Carlo distributions include the number of particles and their weights.

In addition to the equilibrium fields, plasma profiles, fast-ion distribution function and cold neutral density profiles for passive simulations, FIDASIM requires information on the neutral beam geometry, detector geometry, atomic tables, and numerical parameters.

The geometry of the active beam is specified using the conventions of the TRANSP NUBEAM code [40]. Parameters include grid and aperture dimensions, beam focusing and divergence, injection energy and power, and species mix (full, half, and one-third energy current fractions). The present version of the code only supports a single active beam per simulation. To simulate FIDA spectra or NPA signals with multiple active beams, simulation results from multiple simulations are added. This approach is possible without any loss of generality since a local impact of NBI on plasma parameters is negligible, given very short equilibration times along field lines. Similarly, re-absorption of the beam emission radiation by neutrals from other beams is negligible given that plasmas are optically thin.

The NPA detector geometry is specified by detector and aperture shape, size, and orientation (in Cartesian coordinates) for each NPA channel. The spectroscopic geometry is specified by the lens (or mirror) position and optical axis of each sightline (in Cartesian coordinates). 
The final category of input data are the settings for the simulation. These include simulation switches to exercise various code options, the number of Monte Carlo markers to use in different parts of the simulation, the dimensions of the simulation grids, and the wavelength grid for storage of the spectroscopic output.

\section{B Simulation grid}

FIDASIM uses two different simulation grids. First, a Cartesian grid is used to store calculated neutral densities consisting of the injected beam neutrals, the direct charge exchange (DCX) neutrals and halo neutral population. Arbitrary orientations are supported, but the Cartesian grid is usually aligned either with the active beam or with machine coordinates. Inside this grid the Monte Carlo markers, describing bundles of neutrals, are tracked given an initial starting point and velocity vector. Hereby, the times spent in each individual grid cell $t_{\text {cell }}$ are determined which are important when calculating the density contribution to a given cell.

Secondly, a cylindrical grid in $(R, \phi, z)$ coordinates is defined. This grid is used to store and interpolate input data needed for the simulation consisting of the equilibrium magnetic and electric fields, the fast-ion distribution function, and the cold neutral density and kinetic plasma profiles. In case of axisymmetric experiments, the toroidal angle $\phi$ is neglected, while data can be specified over a range of toroidal angles for non-axisymmetric devices such as stellarators. The code uses a 2D interpolation (3D interpolation for nonaxisymmetric devices) to finally obtain the input data at a given $R, \phi, z$ position. This was different in the original version of FIDASIM where all inputs were mapped onto the Cartesian grid. This worked well when calculating relatively localized active NPA and FIDA signals on small grid-sizes. However, when calculating passive signals, the input data must be specified everywhere along a given line of sight. This requires large Cartesian grid sizes and correspondingly, a lot of memory. By instead using the cylindrical grid, the memory demand could be reduced. In addition, the new interpolation scheme provides more accurate profile data, since previously constant profiles were considered inside a given grid-cell.

\section{Injected beam neutrals}

Conceptually, the calculation of the injected neutral densities is the same as in the original version of FIDASIM. Using the known beam geometry, a Monte Carlo procedure launches rays from random positions on the beam source towards the horizontal and vertical focal

point of the beam. Based on the specified beam divergence, the beam is widened by a Gaussian distribution of additional velocity components. Rays that clear the beam aperture(s) are followed into the plasma. The divergence of positive NBI sources typically decreases with the beam energy [41] such that FIDASIM can be supplied with individual divergences 
for the full, half, and one-third energy component of positive beams. All neutrals are emitted from the source in the ground state. Through solution of the collisional-radiative equations, the densities of the full, half, and one-third neutrals (each as a function of energy level $n$ ) are added to the structure that describes each cell. The spectrum of light emitted by the injected beam [often called beam emission spectroscopy (BES) light] is also calculated.

\section{Spectrum routine}

The collisional-radiative module described in 2.1 provides the number of Balmer- $\alpha$ photons emitted by a marker within a given grid cell. In case the grid cell is intersected by a line of sight (LOS), the spectral emission from the marker is added to the synthetic spectrum of the line of sight. The Stark and Doppler shifts of emitted photons are determined by the local electric and magnetic fields, the velocity of the neutral, and the viewing geometry. Emitted photons are Doppler shifted to the wavelength $\lambda=\lambda_{0}\left(1+v_{\|} / c\right)$, then the light is split into 15 Stark components based on the transition probabilities in [42]. Here, $\lambda_{0}$ is the rest Balmer- $\alpha$ wavelength, $c$ is the speed of light, $v_{\|}$is the parallel component of the neutral velocity along the diagnostic LOS, and the electric field in the neutral frame is $\mathbf{E}_{l a b}+\mathbf{v} \times \mathbf{B}$, with the local electric field vector in the laboratory frame, $\mathbf{E}_{l a b}$, the velocity

vector, $\mathbf{v}$, and the magnetic field vector, $\mathbf{B}$. Each marker and cell along a given line of sight therefore contribute with 15 discrete wavelengths to the overall spectrum of this line of sight. Since typically thousands of markers are being simulated, a smooth spectrum can be obtained.

\section{E Fast neutrals}

The calculation of FIDA signals is analogous to the calculations of beam emission calculation, as well as to the calculation of DCX, and halo light described in section 2.2. The only difference is in the generation of the initial fast neutral population. The basic approach is unchanged from the original version of FIDASIM.

The fast-ion distribution function can be input in one of three forms: A guiding center distribution function, a guiding center Monte Carlo distribution, and a full-orbit Monte Carlo distribution. In case a full-orbit Monte Carlo distribution is used as input to FIDASIM, weighted markers are iteratively selected and the energy-level resolved probability of charge exchange with the appropriate neutral population (or populations) is computed. With the cross-section weighted initial flux in hand, the neutrals are followed on their trajectories, evolving according to the collisional-radiative model, while emitting FIDA light.

In the case a guiding-center distribution is provided to FIDASIM, such as the ones generated by TRANSP NUBEAM [40], inverse cumulative distribution function (CDF) sampling 
in the energy and pitch coordinates is used to obtain guiding-center velocity vectors from the distribution function. (The number of markers per cell is governed by the product of fast-ion and neutral density.) Next, a random gyroangle is chosen to complete specification of the velocity $\mathbf{v}$. With $\mathbf{v}$ selected and $\mathbf{B}$ known from the equilibrium inputs, a calculation of the gyroradius $\rho$ gives the actual particle position. The original version of FIDASIM only calculated $\rho$ to leading order, but discrepancies with FIDA data from the low-field spherical tokamak National Spherical Toroidal Experiment (NSTX) [43] motivated a more careful treatment. The current version of FIDASIM includes the higher order corrections to $\rho$ given in Appendix A of [44].

With the position and velocity of the marker established, the charge-exchange probability with the appropriate neutral population (or populations) is computed. Then the same routines as used for the beam and halo neutrals are applied to compute the emitted FIDA spectra or NPA fluxes.

\section{F Atomic rates and cross sections}

The following processes are considered in FIDASIM for hydrogenic neutrals $(\mathrm{H})$ taking into account the initial principal quantum state $n$ and a final principal quantum state $m$ :

$$
\begin{array}{cr}
\mathrm{e}^{-}+\mathrm{H}(n) \longrightarrow \mathrm{e}^{-}+\mathrm{H}^{+}+\mathrm{e}^{-} & \begin{array}{r}
\text { electron impact ionization } \\
\mathrm{e}^{-}+\mathrm{H}(n) \longrightarrow \mathrm{e}^{-}+\mathrm{H}(m)
\end{array} \\
\mathrm{H}^{+}+\mathrm{H}(n) \longrightarrow \mathrm{H}(m)+\mathrm{H}^{+} & \text {electron impact (de-)excitation } \\
\mathrm{H}^{+}+\mathrm{H}(n) \longrightarrow \mathrm{H}^{+}+\mathrm{H}^{+}+\mathrm{e}^{-} & \text {proton charge exchange } \\
\mathrm{H}^{+}+\mathrm{H}(n) \longrightarrow \mathrm{H}^{+}+\mathrm{H}(m) & \text { proton impact (de-)excitation } \\
\mathrm{I}^{Z+}+\mathrm{H}(n) \longrightarrow \mathrm{I}^{(Z-1)+}+\mathrm{H}^{+} & \text {impurity charge exchange } \\
\mathrm{I}^{Z+}+\mathrm{H}(n) \longrightarrow \mathrm{I}^{Z+}+\mathrm{H}^{+}+\mathrm{e}^{-} & \text {impurity impact ionization } \\
\mathrm{I}^{Z+}+\mathrm{H}(n) \longrightarrow \mathrm{I}^{Z+}+\mathrm{H}^{+}(m) & \text { impurity impact (de-)excitation } \\
\mathrm{H}(n) \stackrel{n>m}{\longrightarrow} \mathrm{H}(m)+\gamma & \text { spontaneous de-excitation }
\end{array}
$$

Here, $\mathrm{I}^{\mathrm{Z}+}$ describes an impurity species with atomic number $Z$ such as boron or carbon. The probability of spontaneous de-excitation is given by the Einstein coefficient $[45,46]$, and a photon $\gamma$ is emitted.

To consider these processes for atomic states up to $n=6$, detailed information of the underlying cross sections is required as discussed in the following. Hereby, it should be noted that FIDASIM only treats principal quantum numbers $n$, not angular momentum 
states $l$. The code implicitly assumes that strong fine-structure mixing assures an equilibrium distribution of $l$ states. However, even if this assumption is not strictly valid, it only impacts the calculated Stark splitting (not the overall light intensity); since Doppler shifts are usually much larger than Stark shifts, the impact is minor.

\section{F.1 Charge exchange}

Some of the most important cross sections in FIDASIM are the cross sections for charge exchange. Charge exchange contributes, on the one hand, to the attenuation/ionization of injected neutrals and, on the other hand, to the neutralization of fast and thermal ions. In particular, for the neutralization rates, unbundled cross sections are needed which depend on the initial state $n$ and the final atomic state $m$ :

$$
H^{+}+H(n) \rightarrow H(m)+H^{+}
$$

Here, $H(n)$ is a hydrogen neutral in an atomic state $n$ whose electron is transferred to a hydrogen ion that is neutralized. Because charge exchange reactions can directly populate excited states that are responsible for the Balmer-alpha radiation, detailed knowledge of the charge exchange cross sections into higher m-states is crucial to model FIDA emission correctly.

The ADAS database [25] provides cross sections for charge exchange reactions from $n=1$ to $m=1-4$, from $n=2$ to $m=1,2,3$ and from $n=3$ to $m=2-6$. For excited states above $n=3$, we did not find $m$-state resolved cross sections. Only total cross sections are available from a report by Janev and Smith [47]. Nevertheless, it is possible to infer certain cross sections by the reversibility formula (Eq. 15). This formula states that, e.g. the cross section from $n=4$ to $m=1$ can be related to the one from $n=1$ to $m=4$ by considering the sublevels in a given atomic shell:

$$
\sigma_{m n}=\sigma_{n m} \frac{m^{2}}{n^{2}}
$$

By applying the reversibility formula, several cross sections could be reconstructed based on the ADAS data. However, certain cross sections, such as the one from initial $n=4$ to $n=6$, cannot be obtained by this method. Instead we assume that the probability for a charge exchange reaction from an initial state, $n$, to a final state, $m$, decays exponentially with the energy difference between the two states. This allows us to get estimates for the remaining $m$-resolved cross sections. It should be noted that the sum of the $m$-resolved ADAS cross sections in the $n=1$ level does not perfectly match the Janev and Smith data despite ADAS provides information on all relevant m-levels (The mean difference for energies between $1 \mathrm{keV}$ and $100 \mathrm{keV}$ is $3.1 \%$ ). We, hence, scaled the ADAS cross sections to be consistent with the Janev and Smith data. 
Fig. 18 shows the charge-exchange cross section for reactions of neutrals in the ground state $(n=1)$ that will populate different m-levels $(m=1-6)$. The cross-sections are given as a function of collision energy per atomic mass unit, i.e. for hydrogen atoms.
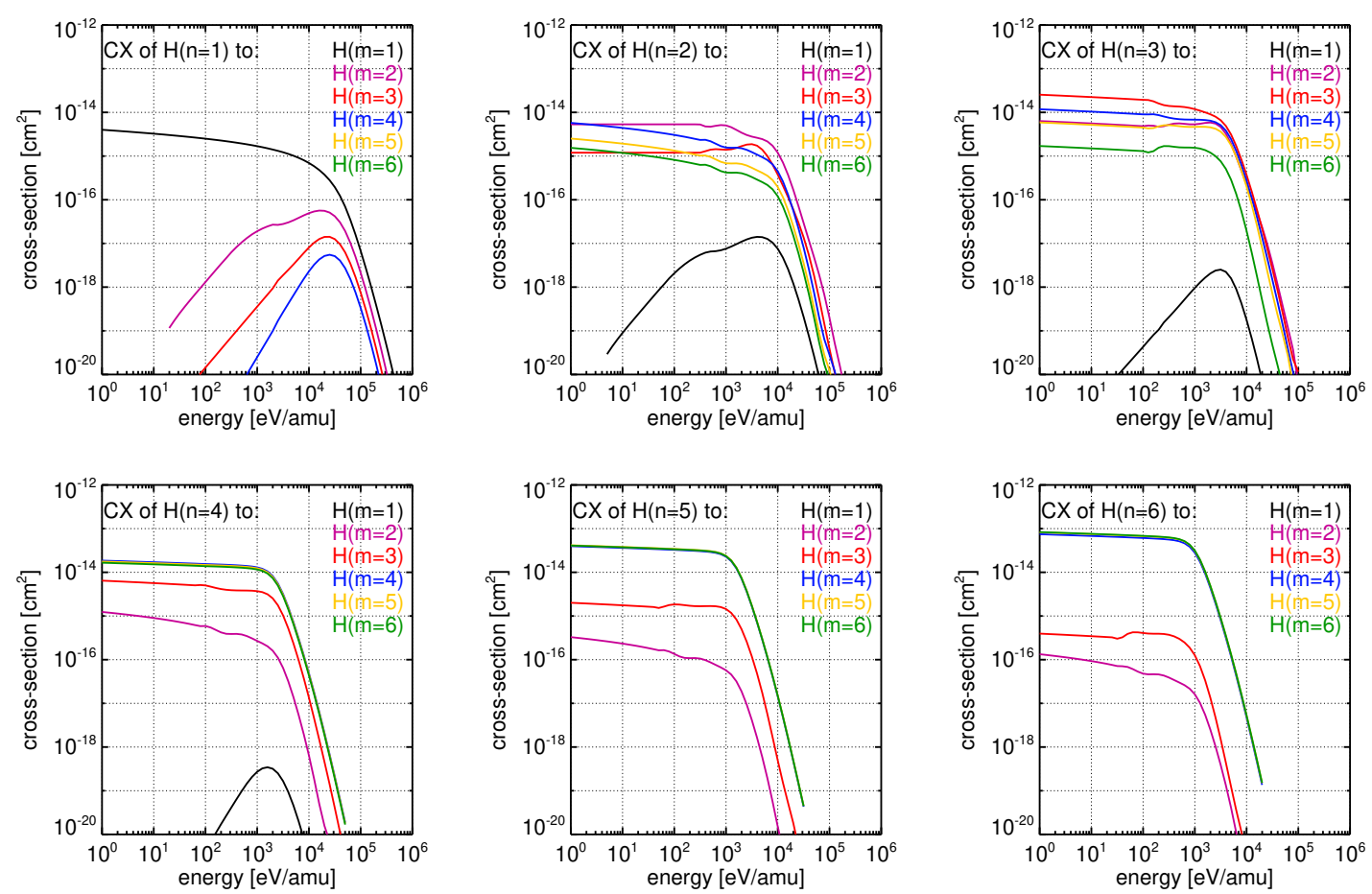

Figure 18: m-level resolved charge-exchange cross sections between hydrogen atoms in the $\mathrm{n}=1-6$ state and hydrogen ions.

For FIDA measurements, the most important cross section is the one from $n=1$ to $m=3$. In equilibrium, most neutrals are in the ground state $(n=1)$, whereas the population of the $n=3$ state is often a factor of 1000 lower. However, the charge exchange cross section from $n=1$ to $m=3$ at $30 \mathrm{keV} / \mathrm{amu}$ is only a factor of 100 lower than that from $n=1$ to $m=1$. The $m=3$ state is, hence, overpopulated after the reaction by $\sim 10$, which quickly decays back to the equilibrium (within a few ns) via the emission of Balmer alpha photons. Consequently, most FIDA radiation is emitted close to the point of neutralization [1], which provides good spatial localization of FIDA measurements.

As already mentioned, those $m$-resolved cross sections are applied when determining the neutralization rates of fast and thermal ions. In addition, the cross sections are needed for the calculation of the attenuation of neutrals. Here, charge exchange processes with impurity ions are also relevant. 


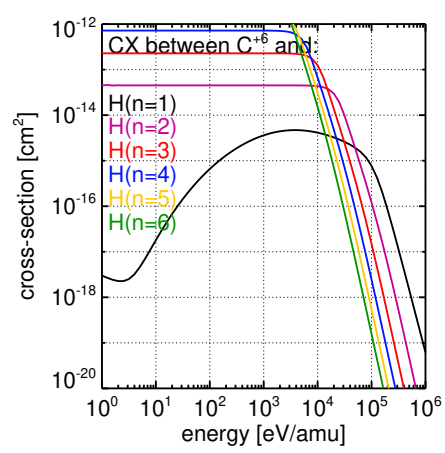

Figure 19: Bundled charge-exchange cross sections between fully stripped carbon ions and hydrogen atoms in the $n=1-6$ state.

Fig. 19, the bundled cross sections for charge exchange reactions between hydrogen neutrals and fully stripped carbon ions. These cross sections for charge exchange reactions are significantly larger than those for collisions with hydrogen ions, explained by the larger charge of the carbon nucleus which attracts electrons more easily. Impurity ions need, hence, to be considered for the attenuation of neutrals in FIDASIM. Cross sections for boron, nitrogen and oxygen are available in FIDASIM and have been taken in part from ADAS and in part from Janev and Smith [47]. However, although different impurities can be selected, the present version of the code only handles a single impurity species which is typically carbon. For carbon, bundled cross-sections from ADAS are for instance available for $\mathrm{n}=1-3$ while scaling formulas from Janev are used for the remaining n-levels.

\section{F.2 Impact ionization}

In addition to the charge exchange reaction, neutrals can be attenuated/ionized by collisions with electrons, hydrogen ions and impurity ions.:

$$
\begin{gathered}
e^{-}+H(n) \rightarrow e^{-}+H^{+}+e^{-} \\
H^{+}+H(n) \rightarrow H^{+}+H^{+}+e^{-} \\
C^{6+}+H(n) \rightarrow C^{6+}+H^{+}+e^{-}
\end{gathered}
$$

Cross sections for collisions with electrons and impurity (carbon) ions are available in a report by Janev [48] and updated, i.e. corrected, cross sections for hydrogen impact ionization have been published by M. O'Mullane [49] for atomic states $n=1-5$. For $n=6$, we used a scaling law provided by Janev and Smith [47] to extrapolate the O'Mullane data. 

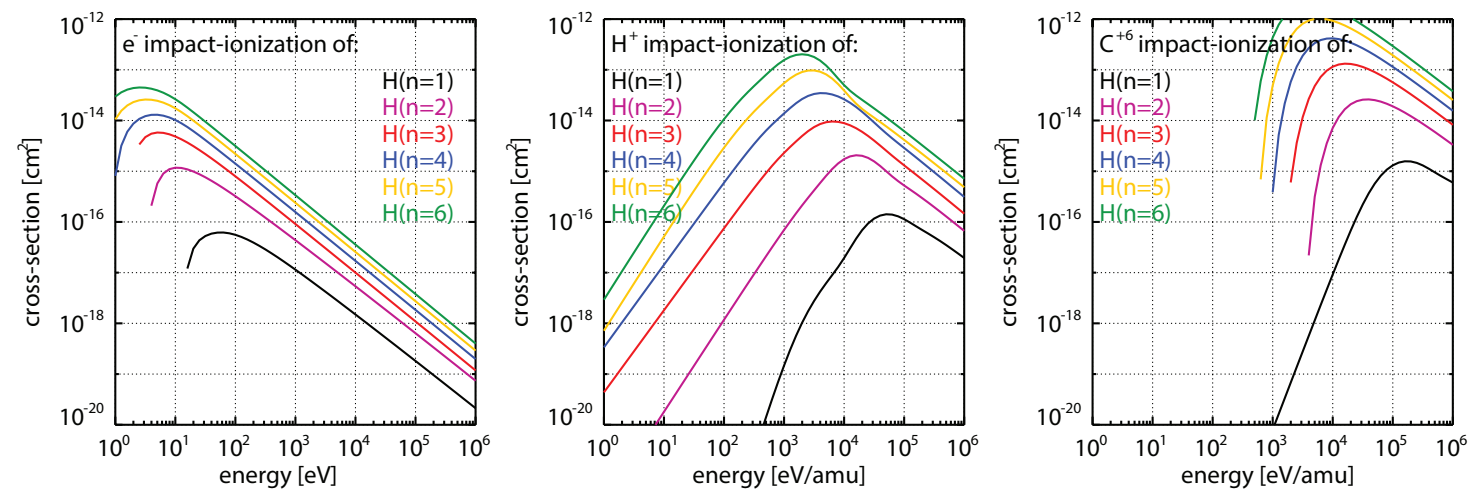

Figure 20: Cross sections for impact ionization of hydrogen neutrals by electrons, hydrogen ions and fully stripped carbon ions.

Fig. 20 illustrates the cross sections for impact ionization of hydrogen neutrals in the atomic states $\mathrm{n}=1-6$ through collisions on electrons, hydrogen ions (protons) and fully stripped carbon ions. For electron impact ionization, the relative collision energy can be approximated by the kinetic energy of the electrons, since the collision energy depends on the relative collision velocity and the reduced mass. The reduced mass $\left(\frac{m_{e} m_{H}}{m_{e}+m_{H}}\right)$ of a collision between an electron and an ion can be approximated by the electron mass $m_{e}$ and, for the collision velocity, the ion velocity can be neglected because electrons are typically much faster. (They are three orders of magnitude lighter than ions.) When comparing the different ionizing cross sections, electron impact ionization is dominant at low collision energies while hydrogen ion impact ionization comes into play at collision energies above $1 \mathrm{keV}$. Depending on the impurity content, carbon impact ionization could also contribute at high collision energies.

\section{F.3 Impact excitation}

Impact excitation is an important mechanism that populates excited states of neutrals that yield Balmer-alpha radiation. In addition, excitation to higher atomic states contributes to the ionization of neutrals because the probability for impact ionization of high levels is very large. Reactions considered in FIDASIM are impact excitation of hydrogen neutrals by electrons (see Fig. 21),

$$
e^{-}+H(n) \rightarrow e^{-}+H(m)
$$

impact excitation by hydrogenic ions (see Fig. 22),

$$
H^{+}+H(n) \rightarrow H^{+}+H(m),
$$

and impact excitation by impurities such as carbon (see Fig. 23),

$$
C^{6+}+H(n) \rightarrow C^{6+}+H(m) .
$$


Cross sections for excited levels up to $n=6$ are considered in FIDASIM. The cross sections for the impact excitation by hydrogen ions and by electrons are taken from [48], while those for impurity impact excitation are based on [47]. Scaling laws are provided by [47], which allow us to calculate cross sections up to $n=12$ or higher. However, we only consider excited states up to $n=6$ in FIDASIM. Because neutrals that are excited into high states are rapidly lost (owing to high cross sections for impact ionization and charge exchange for highly excited states), impact excitation into higher states than $n=6$ are considered a loss mechanism [9]. The corresponding summed cross-sections for excitation into states higher than $n=6$ are shown in Figures 21, 22 and 23 with black dashed lines.
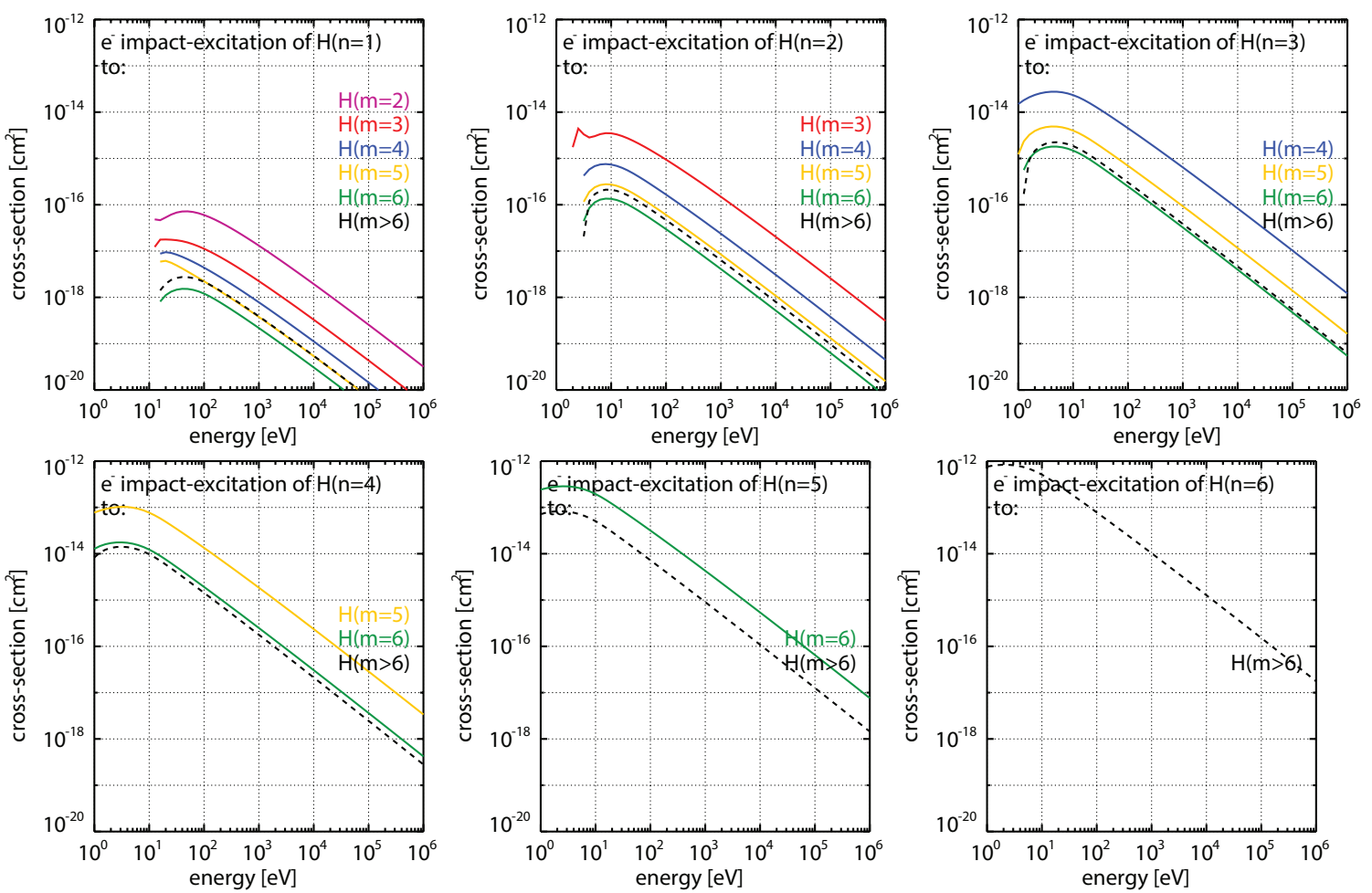

Figure 21: Cross sections for electron impact excitation of hydrogen neutrals of levels $\mathrm{n}=1-6$.

It should be noted that collisional de-excitation is also considered in FIDASIM. To determine cross sections for this process, we apply the reversibility formula, Eq. 15.

\section{F.4 Reaction rates}

A given neutral that moves through the plasma collides with the background plasma (hydrogen, impurities, and electrons) whose velocities can be described by a Maxwell distri- 

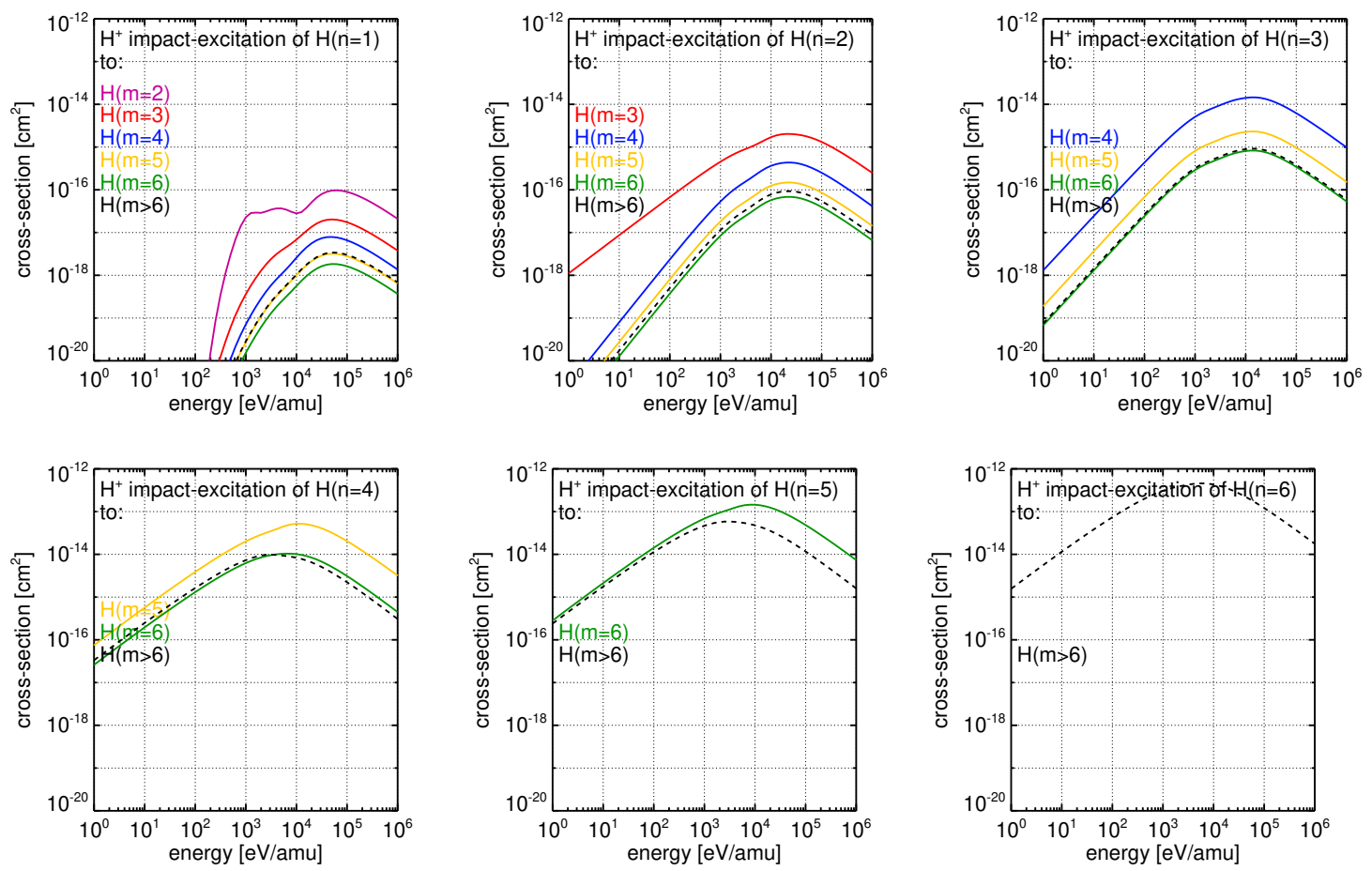

Figure 22: Cross sections for carbon impact excitation of hydrogen neutrals of levels n=1-6.

bution. To consider these collisions, FIDASIM uses pre-calculated tables with effective rate coefficients $\langle\sigma v\rangle_{n m}$ in $\left[\mathrm{cm}^{3} / \mathrm{s}\right]$ that depend on the $n$ and $m$ state, the temperature and on the neutral's energy in the plasma frame of reference (i.e. subtracting the plasma rotation from the neutrals velocity vector). The tables with the effective rate coefficients are calculated by determining the mean value of the product of the energy dependent cross section $\sigma$ and the relative collision velocities between a given projectile (a neutral) and a Maxwell distribution. Per energy and temperature and for each $n$ and $m$ state, a Maxwell distribution of $3 \mathrm{D}$ velocity vectors is defined using random numbers of a normal (Gaussian) distribution that represent a given temperature. The relative collision energy and velocity $v_{r e l}$ with the neutral of the specified energy is then calculated for each velocity vector of the Maxwell distribution. Thus, the corresponding energy-dependent cross-section, and hence, $\sigma v_{r e l}$ can be determined. By taking the mean value of all $\sigma v_{r e l}$ values of the Maxwell distribution, $\langle\sigma v\rangle$ is obtained. Since calculating the $\sigma v_{r e l}$ values for a larger number of velocity vectors is necessary to obtain good statistics, this calculation is time-consuming. FIDASIM therefore uses pre-calculated and tabulated $\langle\sigma v\rangle$ values.

The reaction rates $c_{n m}$ in $[1 / \mathrm{s}]$ can finally be calculated inside the collisional radiative 

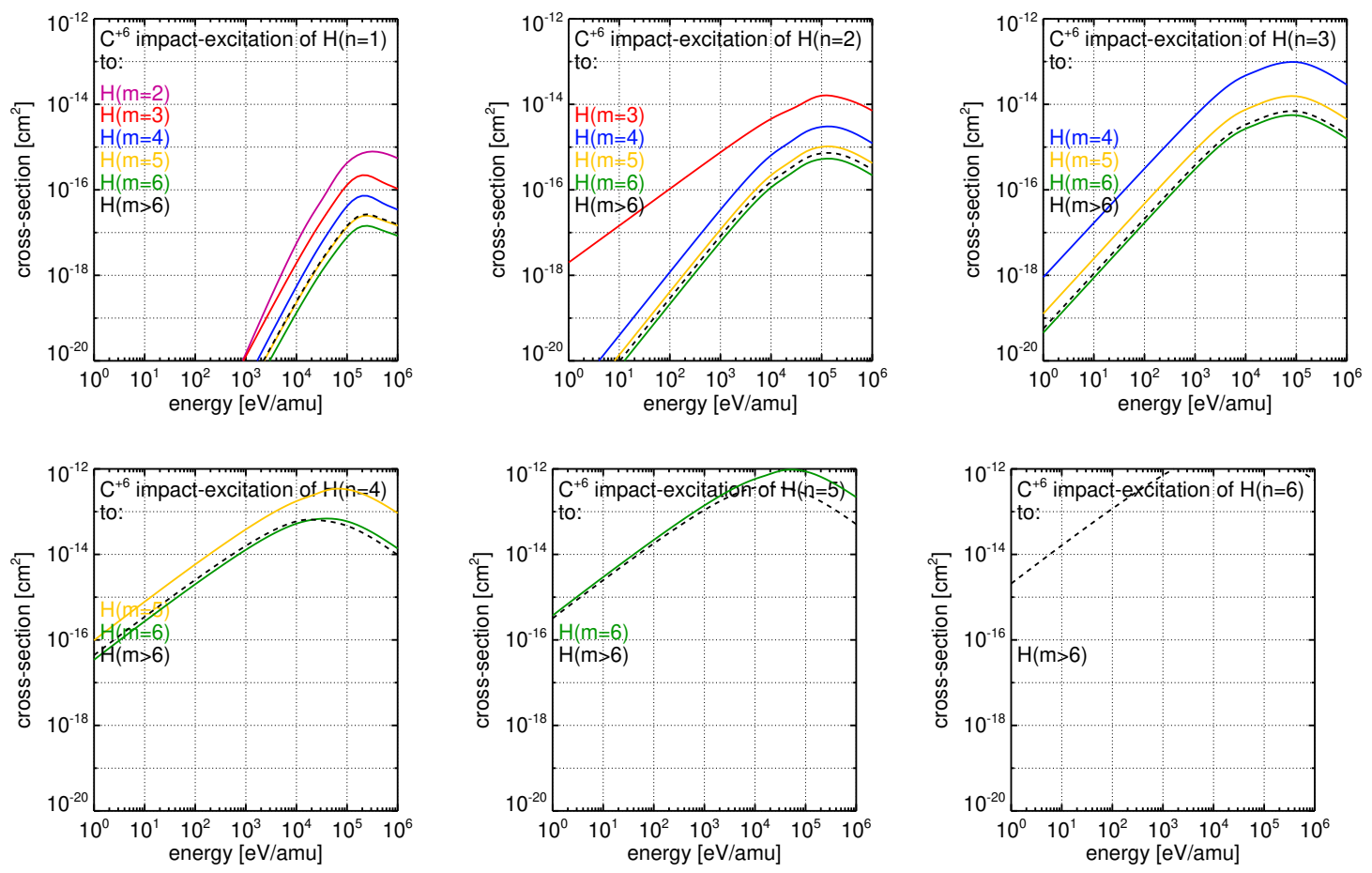

Figure 23: Cross sections for carbon impact excitation of hydrogen neutrals of levels n=1-6.

model of FIDASIM using

$$
c_{n m}=\langle\sigma v\rangle_{n m} \cdot d_{\mathrm{T}}
$$

where $d_{\mathrm{T}}$ is the density of the target particles $\left[\mathrm{cm}^{-3}\right]$.

Fig. 24 illustrates reaction rates as a function of the energy of deuterium neutrals that move through a deuterium plasma featuring an electron density of $5 \times 10^{13} \mathrm{~cm}^{-3}$, an ion and electron temperature of $3 \mathrm{keV}$ and an effective charge of 1.5 with carbon as the only impurity species. In Fig. 24a, rates for the ionizing processes are plotted. Clearly, the charge exchange reaction yields the strongest attenuation of deuterium neutrals, while deuterium impact ionization becomes dominant above $85 \mathrm{keV}$. At energies below $40 \mathrm{keV}$, electron impact ionization becomes important. Because the charge exchange reaction does not attenuate the halo neutrals (a new halo neutral is born by charge exchange), electron impact ionization is particularly important for the halo population. Fig. 24b illustrates the rates for impact excitation from the $n=1$ level into the $m=3$ level, which is relevant for the Balmer-alpha emission. Here, both electron impact and deuterium impact excitation play important roles. The rate for carbon impact excitation, in contrast, is one order of magnitude lower. The rates for the collision-induced de-excitation from the $n=3$ 

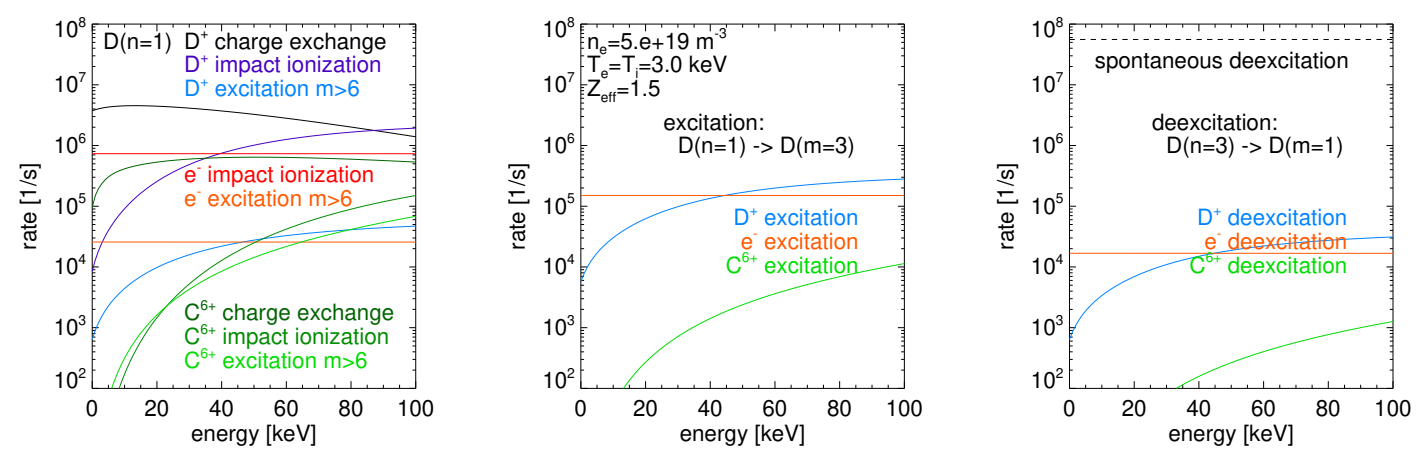

Figure 24: Rates for ionization, excitation and de-excitation of deuterium neutrals for $n_{e}=5 \times 10^{13} \mathrm{~cm}^{-3}, T_{e}=T_{i}=3 \mathrm{keV}$ and $Z_{\text {eff }}=1.5$.

level into the ground state $(m=1)$ are plotted in Fig. 24c. In addition, the rate for spontaneous de-excitation (the Einstein coefficient) is shown with a horizontal dashed line, as the Einstein coefficient does not depend on the deuterium energy. Clearly, spontaneous de-excitation is dominant since the other processes are more than three orders of magnitude less important.

\section{References}

[1] W. W. Heidbrink, "Fast-ion d-alpha measurements of the fast-ion distribution (invited)," Review of Scientific Instruments, vol. 81, no. 10, p. 10D727, 2010.

[2] L. A. Artsimovich, V. V. Afrosimov, I. P. Gladkovskij, S. V. Mirnov, M. P. Petrov, and V. S. Strelkov, "Plasma physics and controlled nuclear fusion research 1965," vol. 2, (Vienna), p. 595, IAEA, 1966.

[3] W. Heidbrink, D. Liu, Y. Luo, E. Ruskov, and B. Geiger, "A code that simulates fastion d-alpha and neutral particle measurements," Commun. Comput. Phys., vol. 10, no. 10, pp. 716-741, 2011.

[4] B. A. Grierson, K. H. Burrell, W. W. Heidbrink, M. J. Lanctot, N. A. Pablant, and W. M. Solomon, "Measurements of the deuterium ion toroidal rotation in the diii-d tokamak and comparison to neoclassical theory," Physics of Plasmas, vol. 19, no. 5, p. $056107,2012$.

[5] S. R. Haskey, B. A. Grierson, L. Stagner, et al., "Active spectroscopy measurements of the deuterium temperature, rotation, and density from the core to scrape off layer on the diii-d tokamak (invited)," Review of Scientific Instruments, vol. 89, p. 10D110, 2018 . 
[6] P. Cano-Megias, E. Viezzer, A. J. van Vuuren, M. Cavedon, D. Cruz-Zabala, R. Dux, B. Geiger, M. Garcia-Munoz, and R. Chacartegui, "Feasibility study for an edge main ion charge exchange recombination spectroscopy system at ASDEX upgrade," Journal of Instrumentation, vol. 14, pp. C10040-C10040, oct 2019.

[7] R. M. McDermott, R. Dux, T. Putterich, et al., "Evaluation of impurity densities from cer spectrscopy measurements at aug," Plasma Physics and Controlled Fusion, vol. 60, p. 095007, 2018.

[8] https://d3denergetic.github.io/FIDASIM/.

[9] I. H. Hutchinson, "Excited-state populations in neutral beam emission," Plasma Physics and Controlled Fusion, vol. 44, no. 1, p. 71, 2002.

[10] http://www.efunda.com/math/ode/syslinearode1.cfm (2012).

[11] X. Du, M. V. Zeeland, W. Heidbrink, and D. Su, "Development and verification of a novel scintillator-based, imaging neutral particle analyzer in DIII-d tokamak," Nuclear Fusion, vol. 58, p. 082006, jun 2018.

[12] M. Salewski, B. Geiger, D. Moseev, W. W. Heidbrink, A. S. Jacobsen, S. B. Korsholm, F. Leipold, J. Madsen, S. K. Nielsen, J. Rasmussen, M. Stejner, and M. Weiland, "On velocity-space sensitivity of FIDA spectroscopy," Plasma Physics and Controlled Fusion, vol. 56, p. 105005, 2014.

[13] M. Salewski, B. Geiger, A. Jacobsen, I. Abramovic, S. Korsholm, F. Leipold, B. Madsen, J. Madsen, R. McDermott, D. Moseev, S. Nielsen, M. Nocente, J. Rasmussen, M. Stejner, M. Weiland, and and, "Deuterium temperature, drift velocity, and density measurements in non-maxwellian plasmas at ASDEX upgrade," Nuclear Fusion, vol. 58, p. 036017, feb 2018.

[14] L. Stagner and W. W. Heidbrink, "Action-angle formulation of generalized, orbitbased, fast-ion diagnostic weight functions," Physics of Plasmas, vol. 24, no. 9, p. $092505,2017$.

[15] M. Salewski, B. Geiger, A. Jacobsen, M. García-Muñoz, W. Heidbrink, S. Korsholm, F. Leipold, J. Madsen, D. Moseev, S. Nielsen, J. Rasmussen, M. Stejner, G. Tardini, and M. W. and, "Measurement of a $2 \mathrm{~d}$ fast-ion velocity distribution function by tomographic inversion of fast-ion d-alpha spectra," Nuclear Fusion, vol. 54, p. 023005, jan 2014.

[16] B. Geiger, M. Weiland, A. Jacobsen, D. Rittich, R. Dux, R. Fischer, C. Hopf, M. Maraschek, R. McDermott, S. Nielsen, T. Odstrcil, M. Reich, F. Ryter, M. Salewski, P. Schneider, and G. Tardini, "Fast-ion transport and neutral beam current drive in ASDEX upgrade," Nuclear Fusion, vol. 55, p. 083001, jun 2015. 
[17] M. Weiland, B. Geiger, A. S. Jacobsen, M. Reich, M. Salewski, and T. O. and, "Enhancement of the FIDA diagnostic at ASDEX upgrade for velocity space tomography," Plasma Physics and Controlled Fusion, vol. 58, p. 025012, jan 2016.

[18] M. Salewski, B. Geiger, A. Jacobsen, P. Hansen, W. Heidbrink, S. Korsholm, F. Leipold, J. Madsen, D. Moseev, S. Nielsen, M. Nocente, T. Odstrčil, J. Rasmussen, L. Stagner, M. Stejner, and M. W. and, "High-definition velocity-space tomography of fast-ion dynamics," Nuclear Fusion, vol. 56, p. 106024, aug 2016.

[19] M. V. Zeeland, J. Yu, W. Heidbrink, N. Brooks, K. Burrell, M. Chu, A. Hyatt, C. Muscatello, R. Nazikian, N. Pablant, D. Pace, W. Solomon, and M. Wade, "Imaging key aspects of fast ion physics in the DIII-d tokamak," Nuclear Fusion, vol. 50, p. 084002, jul 2010.

[20] L. Stagner, Inference of the fast-ion distribution function. PhD thesis, University of California, Irvine, 2018.

[21] A. Jansen van Vuuren, B. Geiger, A. S. Jacobsen, M. Cavedon, R. Dux, and H. Köhnlein, "An edge fast-ion d-alpha system installed at asdex upgrade," Review of Scientific Instruments, vol. 90, no. 10, p. 103501, 2019.

[22] G. Z. Hao, W. W. Heidbrink, D. Liu, M. Podesta, L. Stagner, R. E. Bell, A. Bortolon, and F. Scotti, "Measurement of the passive fast-ion d-alpha emission on the NSTX-u tokamak," Plasma Physics and Controlled Fusion, vol. 60, p. 025026, jan 2018.

[23] B. Geiger, A. N. Karpushov, B. P. Duval, C. Marini, O. Sauter, Y. Andrebe, D. Testa, M. Marascheck, M. Salewski, P. A. Schneider, and and, "Fast-ion transport in low density l-mode plasmas at TCV using FIDA spectroscopy and the TRANSP code," Plasma Physics and Controlled Fusion, vol. 59, p. 115002, sep 2017.

[24] M. Weiland, R. Bilato, R. Dux, B. Geiger, A. Lebschy, F. Felici, R. Fischer, D. Rittich, M. van Zeeland, and and, "RABBIT: Real-time simulation of the NBI fast-ion distribution," Nuclear Fusion, vol. 58, p. 082032, jul 2018.

[25] H. P. Summers, "The adas user manual, version 2.6," 2004.

[26] S. S. Medley, D. Liu, M. V. Gorelenkova, W. W. Heidbrink, and L. Stagner, "Implementation of a $3 \mathrm{~d}$ halo neutral model in the transp code and application to projected nstx-u plasmas," Plasma Physics and Controlled Fusion, vol. 58, p. 025007, 2016.

[27] R. M. McDermott, A. Lebschy, B. Geiger, C. Bruhn, M. Cavedon, M. Dunne, R. Dux, R. Fischer, A. Kappatou, T. Pütterich, and E. Viezzer, "Extensions to the charge exchange recombination spectroscopy diagnostic suite at asdex upgrade," Review of Scientific Instruments, vol. 88, no. 7, p. 073508, 2017. 
[28] B. A. Grierson, K. H. Burrell, C. Chrystal, R. J. Groebner, D. H. Kaplan, W. W. Heidbrink, J. M. Muñoz Burgos, N. A. Pablant, W. M. Solomon, and M. A. Van Zeeland, "Active spectroscopic measurements of the bulk deuterium properties in the diii-d tokamak (invited)," Review of Scientific Instruments, vol. 83, no. 10, p. 10D529, 2012.

[29] S. R. Haskey, B. A. Grierson, K. H. Burrell, C. Chrystal, R. J. Groebner, D. H. Kaplan, N. A. Pablant, and L. Stagner, "Measurement of deuterium density profiles in the h-mode steep gradient region using charge exchange recombination spectroscopy on diii-d," Review of Scientific Instruments, vol. 87, no. 11, p. 11E553, 2016.

[30] B. LaBombard, "KN1D: A 1-D space kinetic, 2-D velocity, transport algorithm for atomic molecular hydrogen in an ionizing plasma," Research Report PSFC-RR-01-3, Massachusetts Institute of Technology, Plasma Science and Fusion Center, 175 Albany St., Cambridge, MA 02139 USA, 2001.

[31] A. Iiyoshi, A. Komori, A. Ejiri, M. Emoto, H. Funaba, M. Goto, K. Ida, H. Idei, S. Inagaki, S. Kado, O. Kaneko, K. Kawahata, T. Kobuchi, S. Kubo, R. Kumazawa, S. Masuzaki, T. Minami, J. Miyazawa, T. Morisaki, S. Morita, S. Murakami, S. Muto, T. Mutoh, Y. Nagayama, Y. Nakamura, H. Nakanishi, K. Narihara, K. Nishimura, N. Noda, S. Ohdachi, N. Ohyabu, Y. Oka, M. Osakabe, T. Ozaki, B. Peterson, A. Sagara, S. Sakakibara, R. Sakamoto, H. Sasao, M. Sasao, K. Sato, M. Sato, T. Seki, T. Shimozuma, M. Shoji, H. Suzuki, Y. Takeiri, K. Tanaka, K. Toi, T. Tokuzawa, K. Tsumori, K. Tsuzuki, K. Watanabe, T. Watari, H. Yamada, I. Yamada, S. Yamaguchi, M. Yokoyama, R. Akiyama, H. Chikaraishi, K. Haba, S. Hamaguchi, M. Iima, S. Imagawa, N. Inoue, K. Iwamoto, S. Kitagawa, J. Kodaira, Y. Kubota, R. Maekawa, T. Mito, T. Nagasaka, A. Nishimura, C. Takahashi, K. Takahata, Y. Takita, H. Tamura, T. Tsuzuki, S. Yamada, K. Yamauchi, N. Yanagi, H. Yonezu, Y. Hamada, K. Matsuoka, K. Murai, K. Ohkubo, I. Ohtake, M. Okamoto, S. Satoh, T. Satow, S. Sudo, S. Tanahashi, K. Yamazaki, M. Fujiwara, and O. Motojima, "Overview of the large helical device project," Nuclear Fusion, vol. 39, pp. 1245-1256, sep 1999.

[32] M. Osakabe, S. Murakami, M. Yoshinuma, et al., "Fast ion charge exchange spectroscopy measurement using a radially injected neutral beam on the large helical device," Review of Scientific Instruments, vol. 79, p. 10E519, 2008.

[33] Y. Fujiwara, S. Kamio, Y. Yamaguchi, et al., "Fast-ion d alpha diagnostic with enhanced fidasim in the large helical device," Nuclear Fusion, vol. 60, p. submitted, 2020 .

[34] B. Geiger, Fast-ion transport studies using FIDA spectroscopy at the ASDEX Upgrade tokamak. PhD thesis, Ludwig-Maximilians-Universität München, January 2013. 
[35] C. S. Collins, W. W. Heidbrink, M. E. Austin, G. J. Kramer, D. C. Pace, C. C. Petty, L. Stagner, M. A. Van Zeeland, R. B. White, and Y. B. Zhu, "Observation of criticalgradient behavior in alfvén-eigenmode-induced fast-ion transport," Phys. Rev. Lett., vol. 116, p. 095001, Feb 2016.

[36] W. W. Heidbrink, C. S. Collins, M. Podestà, G. J. Kramer, D. C. Pace, C. C. Petty, L. Stagner, M. A. Van Zeeland, R. B. White, and Y. B. Zhu, "Fast-ion transport by alfvén eigenmodes above a critical gradient threshold," Physics of Plasmas, vol. 24, no. 5, p. 056109, 2017.

[37] A. N. Karpushov, B. P. Duval, and C. Schlatter, "Neutral particle analyzer diagnostics on the tcv tokamak," Review of Scientific Instruments, vol. 77, p. 033504, 2006.

[38] B. Geiger, A. N. Karpushov, P. Lauber, S. E. Sharapov, M. Dreval, F. Bagnato, M. Baquero-Ruiz, A. D. Molin, B. P. Duval, M. Garcia-Munoz, C. Marini, M. Nocente, O. Sauter, L. Stipani, D. S. Testa, and M. Vallar, "Observation of alfvén eigenmodes driven by off-axis neutral beam injection in the tcv tokamak," Plasma Physics and Controlled Fusion, 2020.

[39] M. Podestà, W. W. Heidbrink, R. E. Bell, and R. Feder, "The NSTX fast-ion d-alpha diagnostic," Review of Scientific Instruments, vol. 79, p. 10E521, 2008.

[40] A. Pankin, D. McCune, R. Andre, G. Bateman, and A. Kritz, "The tokamak monte carlo fast ion module nubeam in the national transport code collaboration library," Computer Physics Communications, vol. 159, no. 3, pp. 157 - 184, 2004.

[41] H. W. Kugel, G. M. Gammel, L. R. Grisham, R. Kaita, J. H. Kamperschroer, R. A. Langley, C. W. Magee, S. S. Medley, T. J. Murphy, A. L. Roquemore, and M. D. Williams, "Measurements of neutral beam species, impurities, spatial divergence, energy dispersion, pressure, and reionization using the tftr u.s. common long pulse ion source," Review of Scientific Instruments, vol. 60, no. 1, pp. 37-52, 1989.

[42] H. A. Bethe and E. E. Salpeter, Quantum mechanics of one-and two-electron atoms. Springer Science \& Business Media, 2012.

[43] W. Heidbrink, E. Ruskov, D. Liu, L. Stagner, E. Fredrickson, M. Podestà, and A. Bortolon, "Analysis of fast-ion d-alpha data from the national spherical torus experiment," Nuclear Fusion, vol. 56, p. 056005, apr 2016.

[44] E. V. Belova, N. N. Gorelenkov, and C. Z. Cheng, "Self-consistent equilibrium model of low aspect-ratio toroidal plasma with energetic beam ions," Physics of Plasmas, vol. 10, no. 8, pp. 3240-3251, 2003.

[45] A. Einstein, "Zur quantentheorie der strahlung," Physikalische Zeitschrift, no. 18, p. $121-128,1917$. 
[46] W. L. Wiese, M. W. Smith, and B. M. Miles, Atomic Transition Probabilities. NSRDSNBS 22. U.S.A. National Bureau of Standards, Washington DC, 1969.

[47] R. K. Janev and J. J. Smith, "Atomic and plasma-material interaction data for fusion," Nucl. Fusion Suppl. Special Issue, vol. 4, no. 1, p. 172, 1993.

[48] R. K. Janev, D. Reiter, and U. Samm, "Collision processes in low-temperature hydrogen plasmas," B2EIRENE report, 2004.

[49] M. O. Mullane, "Review of proton impact driven ionisation from the excited levels in neutral hydrogen beams," 2009. 